ARTICLE

Received 2 Feb 2013 | Accepted 15 Aug 2013 | Published 20 Sep 2013

DOl: $10.1038 /$ ncomms3443 OPEN

\title{
Targeting BIG3-PHB2 interaction to overcome tamoxifen resistance in breast cancer cells
}

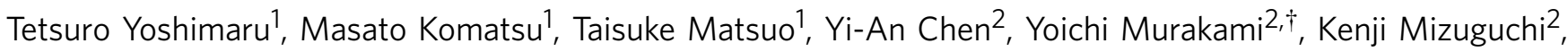 \\ Eiichi Mizohata $^{3}$, Tsuyoshi Inoue ${ }^{3}$, Miki Akiyama ${ }^{4}$, Rui Yamaguchi ${ }^{5}$, Seiya Imoto ${ }^{6}$, Satoru Miyano ${ }^{6}$, \\ Yasuo Miyoshi ${ }^{7}$, Mitsunori Sasa ${ }^{8}$, Yusuke Nakamura ${ }^{4,9}$ \& Toyomasa Katagiri ${ }^{1}$
}

The acquisition of endocrine resistance is a common obstacle in endocrine therapy of patients with oestrogen receptor- $\alpha(E R \alpha)$-positive breast tumours. We previously demonstrated that the BIG3-PHB2 complex has a crucial role in the modulation of oestrogen/ER $\alpha$ signalling in breast cancer cells. Here we report a cell-permeable peptide inhibitor, called ERAP, that regulates multiple $E R \alpha$-signalling pathways associated with tamoxifen resistance in breast cancer cells by inhibiting the interaction between BIG3 and PHB2. Intrinsic PHB2 released from BIG3 by ERAP directly binds to both nuclear- and membrane-associated ER $\alpha$, which leads to the inhibition of multiple ER $\alpha$-signalling pathways, including genomic and nongenomic ER $\alpha$ activation and ER $\alpha$ phosphorylation, and the growth of ER $\alpha$-positive breast cancer cells both in vitro and in vivo. More importantly, ERAP treatment suppresses tamoxifen resistance and enhances tamoxifen responsiveness in ER $\alpha$-positive breast cancer cells. These findings suggest inhibiting the interaction between BIG3 and PHB2 may be a new therapeutic strategy for the treatment of luminal-type breast cancer.

\footnotetext{
${ }^{1}$ Division of Genome Medicine, Institute for Genome Research, The University of Tokushima, 3-18-15, Kuramoto-cho, Tokushima 770-8503, Japan. ${ }^{2}$ National Institute of Biomedical Innovation, 7-6-8 Asagi-Saito, Ibaraki-City, Osaka 567-0085, Japan. ${ }^{3}$ Department of Applied Chemistry, Graduate School of Engineering, Osaka University, 2-1, Yamada-oka, Suita, Osaka 565-0871, Japan. ${ }^{4}$ Laboratory of Molecular Medicine, University of Tokyo, 4-6-1, Shirokanedai, Minato-ku, Tokyo 108-8639, Japan. ${ }^{5}$ Laboratory of Sequence Analysis, The University of Tokyo, 4-6-1, Shirokanedai, Minato-ku, Tokyo 108-8639, Japan. ${ }^{6}$ Laboratory of DNA Information Analysis, Human Genome Centre, Institute of Medical Science, The University of Tokyo, 4-6-1, Shirokanedai, Minato-ku, Tokyo 108-8639, Japan. ${ }^{7}$ Division of Breast and Endocrine Surgery, Department of Surgery, Hyogo College of Medicine, 1-1, Mukogawa-cho, Nishinomiya, Hyogo 663-8501, Japan. ${ }^{8}$ Department of Surgery, Tokushima Breast Care Clinic, 4-7-7, Nakashimada, Tokushima 770-0052, Japan. ${ }^{9}$ Department of Medicine and Surgery, The University of Chicago, 900 E 57th Street, KCBD6126, Chicago, Illinois 60637, USA. † Present address: Graduate School of Information Sciences, Tohoku University, 6-3-09, Aramaki-aza-aoba, Aoba-ku, Sendai-city, Miyagi 980-8579, Japan. Correspondence and requests for materials should be addressed to T.K. (email: tkatagi@genome.tokushima-u.ac.jp).
} 
$\mathrm{B}$ reast cancer is the most common cancer among women worldwide ${ }^{1,2}$. More than $70 \%$ of primary breast tumours are oestrogen receptor- $\alpha(\mathrm{ER} \alpha)$-positive, and the interactions between oestrogen (E2) and ER $\alpha$ dramatically enhance the proliferative and metastatic activity of breast tumour cells ${ }^{3,4}$. E2 biological actions are mediated by both genomic and nongenomic mechanisms; in the former type nuclear ER $\alpha$ functions as a ligand-dependent transcription factor that regulates target gene expression levels ${ }^{3,5}$, whereas in the latter type E2-bound ER $\alpha$ in the plasma membrane associates with a variety of signalling molecules, including IGF receptor $\beta$ (IGF-1R $\beta$ ), phosphoinositide 3-kinase (PI3K) and $\mathrm{SH} 2$ domain containing (Shc), which results in Akt and mitogen-activated protein kinase (MAPK) activation or increased nuclear ER $\alpha$ phosphorylation ${ }^{6-10}$. Thus, ER $\alpha$ has a pivotal role in the E2 signalling network and therefore represents an important therapeutic target for breast cancer.

The selective ER modulator tamoxifen directly inhibits E2 and $\mathrm{ER} \alpha$ interactions, and is a standard treatment offered to patients with $E R \alpha$-positive breast cancer ${ }^{11-13}$. Nonetheless, tumours often develop resistance, leaving patients with recurrent tumours that lack targeted therapeutic options ${ }^{14,15}$. The potential mechanisms for either intrinsic or acquired endocrine resistance remain poorly understood, but they clearly include $\mathrm{ER} \alpha$-coregulatory proteins and cross-talk between the ER $\alpha$ pathway and other growth factors and kinase networks ${ }^{10,11,16}$. This knowledge has led to numerous treatment strategies combining endocrine and targeted inhibitor therapies ${ }^{17-19}$; however, comprehensive measures for this problem remain unresolved. Therefore, identifying the factors and pathways responsible for resistance and defining ways to overcome it represent important therapeutic challenges in breast cancer research.

The novel E2/ER $\alpha$ signalling regulator brefeldin A-inhibited guanine nucleotide-exchange protein 3 (BIG3), which is exclusively overexpressed in a majority of breast cancers, was recently identified from genome-wide expression profiles $^{20,21}$. BIG3 interacts and colocalizes with prohibitin 2 (PHB2) in the cytoplasm of breast cancer cells ${ }^{20,21}$. PHB2 is known to function as a corepressor of $\mathrm{ER} \alpha^{22,23}$. Our previous study demonstrated that when BIG3 was knocked down by small interfering RNA, E2 stimulation led to the nuclear translocation of a majority of the cytoplasmic PHB2, enhanced the interaction between PHB2 and $\mathrm{ER} \alpha$, and suppressed $\mathrm{ER} \alpha$ transcriptional activity ${ }^{21}$. Accordingly, we hypothesized that BIG3 captures PHB2 in the cytoplasm of cancer cells and thereby inhibits the suppressive ability of PHB2 in the presence of $\mathrm{E} 2$, resulting in the constitutive activation of $\mathrm{ER} \alpha$ signalling pathways.

Here we describe a synthetic, cell-penetrating, dominantnegative peptide that inhibits the $\mathrm{E} 2 / \mathrm{ER} \alpha$ signalling network by activating the tumour suppressive ability of PHB2. This peptide also enhanced tamoxifen responsiveness and anti-tumour effects in tamoxifen-resistant (TAM-R) breast cancers. Thus, the regulation of E2 signalling by targeting the BIG3-PHB2 interaction introduces a new potential therapeutic approach for endocrine-resistant tumours, as well as ER $\alpha$-positive breast cancers.

\section{Results}

Identification of the BIG3-PHB2 interacting region. Previous studies have shown that the BIG3-PHB2 complex has a critical role in breast cancer cell growth ${ }^{21}$, and strategies capable of inhibiting this interaction may represent novel therapies for breast cancer. Therefore, we first attempted to determine the BIG3 region(s) required for the interaction with PHB2 through in silico and biochemical analyses. First, we independently cotransfected five partial constructs of FLAG-tagged BIG3 (Fig. 1a) with HA-tagged PHB2 (HA-PHB2) into COS-7 cells. Immunoprecipitation with an anti-FLAG antibody indicated that HAPHB2 co-immunoprecipitated with BIG3 $_{1-434}, \mathrm{BIG}_{1-250}$ and full-length BIG3 (Fig. 1b), suggesting that the 101-250th amino acid region of BIG3 is minimally required for its interaction with PHB2.

In parallel with this approach, we attempted to predict the protein binding sites on BIG3 using the PSIVER (Protein-protein interaction SItes prediction server) software ${ }^{24}$, and we identified a cluster of candidate binding residues within the 101-250th amino acid region. This cluster region contained three of the highest scoring ( $\geq 0.6)$ residues (Q165, D169 and Q173; Fig. 1c), which were oriented in the same direction (Fig. 1d). Indeed, the BIG3 mutations in which all of these target residues were substituted with alanine almost completely abolished the interaction with HA-PHB2 (Fig. 1e), indicating the importance of Q165, D169 and Q173 for BIG3 heterodimerization with PHB2. Moreover, D169 was the most critical site among these residues for binding, although an alanine mutation on each residue resulted in reduced binding (Supplementary Fig. S1). Accordingly, we focused on these residues as candidate PHB2-binding residues.

A peptide with dominant-negative influence on $\mathrm{ER} \alpha$ activity. We next investigated the possibility of a cell-penetrating peptide as a dominant-negative inhibitor targeting the BIG3-PHB2 interaction, and designed a specific peptide that included these PHB2-binding residues to target the BIG3-PHB2 interaction. This peptide, referred to as $\mathrm{ER} \alpha$ activity-regulator synthetic peptide (ERAP), contained the BIG3 potential binding residues (165-QMLSDLTLQLRQR-177) and membrane-permeable polyarginine residues (11R) at its $\mathrm{NH}_{2}$ terminus (Fig. 2a). As negative controls, peptides containing a scrambled amino acid sequence (scrERAP) and either alanine mutations at key residues (mtERAP) were constructed (Fig. 2a). Indeed, co-immunoprecipitation experiments revealed that ERAP, but not mtERAP or scrERAP, completely inhibited the complex formation of endogenous BIG3 and PHB2 in the ER $\alpha$-positive breast cancer cell lines MCF-7 and KPL-3C, which strongly express BIG3 and PHB2 (Fig. 2b and Supplementary Fig. S2). We also examined the direct inhibition of the BIG3-PHB2 interaction using ERAP. As expected, HA-ERAP bound to His-tagged recombinant PHB2 protein and inhibited the BIG3-PHB2 interaction in a dosedependent manner, whereas scrERAP did not (Fig. 2c). In addition, mtERAP exhibited modest binding to the PHB2 protein at levels substantially lower than ERAP (Fig. 2c). Surface plasmon resonance (BIAcore) interaction analysis revealed that ERAP bound to the His-tagged recombinant PHB2 with a dissociation constant $(\mathrm{Kd})=18.9 \mu \mathrm{M}$ (Fig. $2 \mathrm{~d}$ ). Thus, our data suggested that ERAP directly bound to PHB2, resulting in the specific inhibition of BIG3-PHB2 complex formation.

ERAP translocates PHB2 and attenuates nuclear ER $\alpha$ activation. We investigated the subcellular distribution of endogenous PHB2 in breast cancer cells following ERAP treatment by immunocytochemical and biochemical approaches. In the presence of E2, treatment with ERAP, but not with scrERAP, led to a significant increase in the amount of nuclear PHB2 in a timedependent fashion (Fig. 3a). In addition, in the presence of E2, ERAP treatment led to a decrease in cytoplasmic PHB2, thereby substantially increasing the interaction between PHB2 and ER $\alpha$ in the nucleus even after $1 \mathrm{~h}$ (Fig. 3b). Furthermore, ERAP coimmunoprecipitated and colocalized with endogenous PHB2 in the nucleus and the cytoplasm (Supplementary Fig. S3a,b) but did not directly bind to ER $\alpha$ or BIG3. These findings suggested that ERAP caused PHB2 to be released from BIG3 and led to 
a

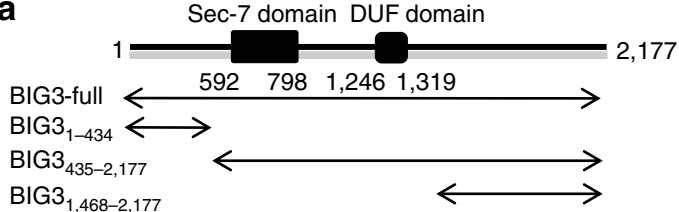

C

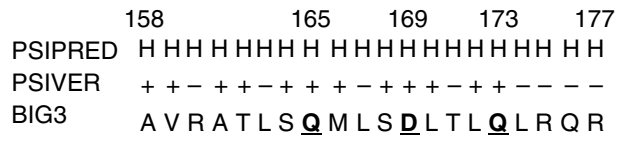

d

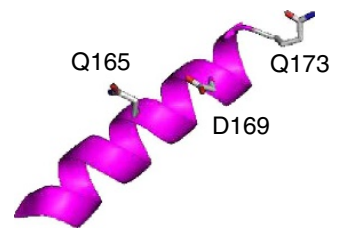

b
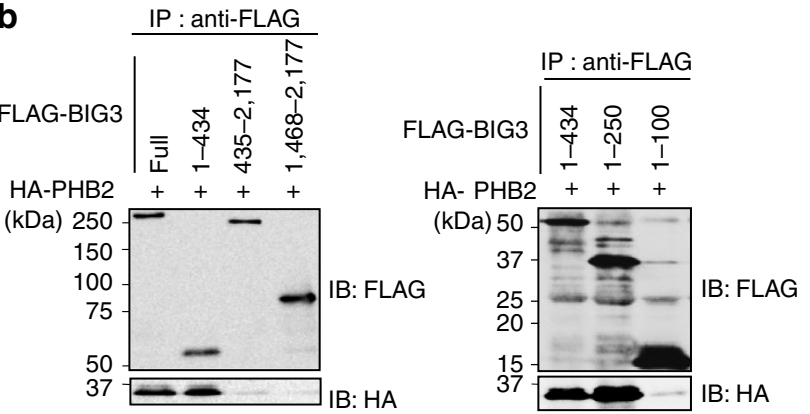

e

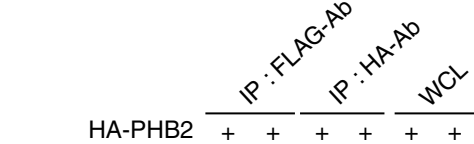

$+-+-++$

FLAG-BIG3 mutant $-+\quad+\quad+$
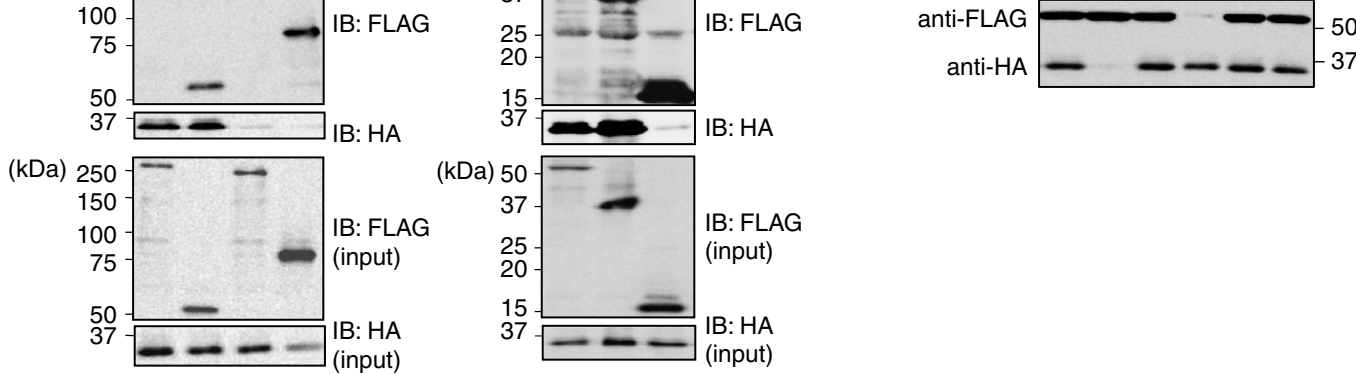

Figure 1 | Identification of the BIG3-PHB2 interacting region. (a) The schematic representation of human BIG3 and the five FLAG-BIG3 partial clones lacking one of the terminal regions is shown. (b) Immunoblot analyses were performed to identify the PHB2-binding region in BIG3. COS-7 cells were transfected with the indicated BIG3 constructs (full-length BIG3, BIG3 $1-434, B I G 3_{435-2,177}, B I G 3_{1,468-2177}, B I G 3_{1-100}$ and BIG3 $1-250$ ) and HA-PHB2.

After $48 \mathrm{~h}$, the cells were lysed and FLAG-BIG3 was immunoprecipitated with an anti-FLAG antibody. Immunoprecipitated proteins and a portion of the original cell lysates (input) were immunoblotted as indicated. (c) The predicted interaction sites, as determined using PSIVER software, are shown. The underlined bold letters indicate the residues most likely to be involved in BIG3-PHB2 binding. (d) The putative PHB2-binding sites (Q165, D169 and Q173) on a predicted three-dimensional structure of BIG3 protein are shown. (e) Immunoblots were performed to assess the PHB2-binding region in BIG3 protein. The lysates from COS-7 cells transfected with BIG3 ${ }_{1-434}$ or mutant BIG3 constructs were immunoprecipitated with anti-FLAG and anti-HA antibodies to detect BIG3 and PHB2, respectively. Full-length images of immunoblots are shown in Supplementary Fig. S9.

E2-dependent PHB2 nuclear translocation, eventually resulting in the interaction of PHB2 with nuclear ER $\alpha$ in cancer cells.

$\mathrm{ER} \alpha$ has been shown to modulate transcription in two ways: (i) through direct binding to oestrogen-responsive elements (EREs) located in the promoter and/or enhancer regions of target genes ${ }^{25}$ and (ii) by serving as a co-activator of other transcription factors such as AP-1 (ref. 26). Therefore, we explored the impact of ERAP treatment on these two modes of ER $\alpha$ transcriptional activity. First, we performed a chromatin immunoprecipitation (ChIP) assay with E2-stimulated MCF-7 cells. The results showed that ERAP treatment induced E2dependent recruitment of the endogenous ER $\alpha-\mathrm{PHB} 2$ complex on the ER $\alpha$ target genes, TFF1 and CCND1, respectively, (Fig. 3c), suggesting that ERAP did not inhibit the ability of ER $\alpha$ to bind ERE or AP-1. In luciferase assays with ERE or AP-1 reporters, ERAP significantly inhibited both forms of E2-induced ER $\alpha$ transcriptional activity in a dose-dependent manner in MCF-7 and KPL-3C cells (Supplementary Fig. S3c), but no significant inhibition was observed with scrERAP or mtERAP. These results indicated that ERAP suppressed ER $\alpha$ transcriptional activity levels through both canonical ERE- and non-canonical AP-1binding mechanisms.

PHB2 is known to act as an ER $\alpha$ transcriptional corepressor by competing with the co-activator SRC-1 to bind $\mathrm{ER} \alpha^{23}$ and by recruiting histone deacetylase 1 (HDAC1; ref. 27) and another corepressor, $\mathrm{NcoR}^{28}$. Thus, we next explored the effect of ERAP on this recruitment in MCF-7 and KPL-3C cells using ChIP assays. Stimulation with E2 alone recruited SRC-1 to $\mathrm{ER} \alpha$, whereas ERAP treatment led to the direct association of PHB2 with ER $\alpha$ in the presence of E2, reduced SRC-1 binding to $\mathrm{ER} \alpha$, and enhanced the recruitment of HDAC1 and NcoR in MCF-7 (Fig. 3d) and KPL-3C cells (Supplementary Fig. S3d). Moreover, we performed a ChIP-quantitative PCR assay, with E2-stimulated MCF-7 cells. The results showed that ERAP treatment significantly reduced the E2-dependent recruitment of endogenous SRC-1 on the TFF1 gene but increased the E2-dependent recruitment of endogenous NcoR, HDAC1 and PHB2 (Supplementary Fig. S3e). In contrast, ERAP treatment had no effect on ChIP assay using an anti-BIG3 antibody (Supplementary Fig. S3e) or on ER $\alpha$ expression at the mRNA or protein level (Supplementary Fig. S3f). Subsequently, we investigated the HDAC activity of PHB2 immunoprecipitates in MCF-7 cells and found that the chromatin-remodelling complexes recruited by ERAP treatment led to a significant increase in HDAC activity (Fig. 3e). Moreover, ERAP significantly suppressed E2-induced expression of TFF1, CCND1, $-M y c$, E2F1 and $P g R^{29-33}$ (Fig. 3f). In addition, we validated the suppressive effect of BIG3 on ER $\alpha$ transcriptional activity. BIG3 

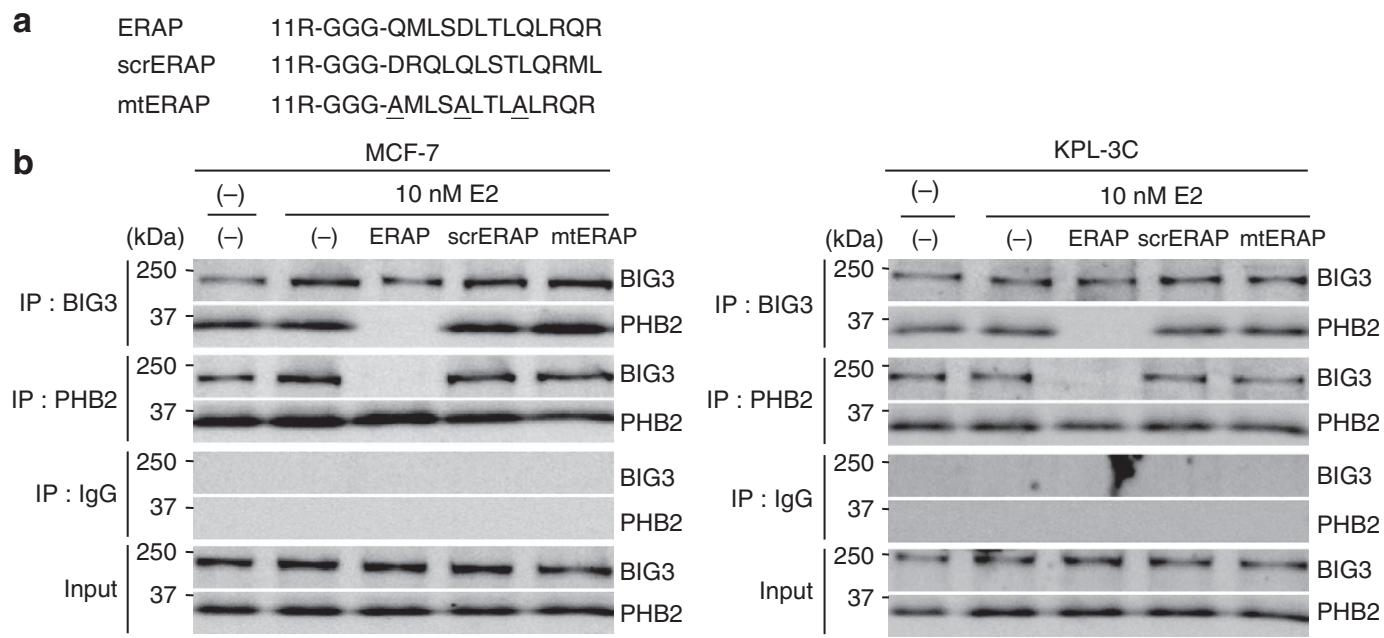

C HA-ERAP $(\mu M) \quad 0 \quad 0 \overline{0.1110100} \frac{0.1110100}{0.1110100}$ Lysate of FLAG-BIG3 -+++++++++++ $10 \mu \mathrm{g}$ His-PHB2 -+++++++++++++

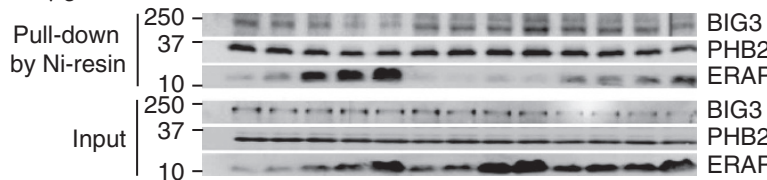

d

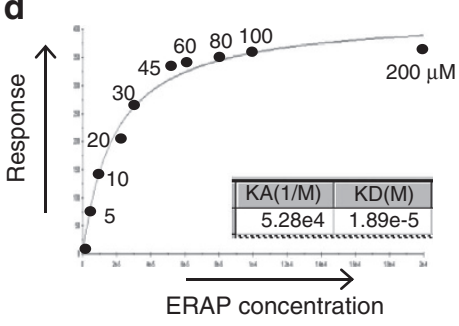

Figure 2 | ERAP inhibits the interaction of BIG3 with PHB2. (a) The ERAP, scrERAP and mtERAP sequences are shown. (b) The inhibitory effects of ERAP treatment on BIG3-PHB2 interactions were evaluated in MCF-7 (left) and KPL-3C cells (right). (c) Direct inhibition of the BIG3-PHB2 interaction by ERAP was evaluated. The lysates of COS-7 cells, transiently transfected with FLAG-BIG3, were incubated with $6 \times$ His-tagged recombinant PHB2 (His-PHB2) and HA-ERAP, HA-scrERAP or HA-mtERAP for $1 \mathrm{~h}$. Then, His-PHB2 was captured with Ni-NTA agarose, and the bound fractions were immunoblotted as indicated. (d) In vitro direct interaction of ERAP and PHB2 was evaluated by BIAcore.

depletion caused a significant reduction in the canonical ERE and non-canonical AP-1 ER $\alpha$ transcriptional activities in ER $\alpha$ positive MCF-7 cells but did not affect ER $\alpha$-negative MDAMB-231 cells (Supplementary Fig. S3g). Taken together, these findings indicated that nuclear-translocated PHB2 following ERAP treatment directly bound to ER $\alpha$ and acted as a corepressor by recruiting HDAC1 and NcoR, thereby leading to an almost complete suppression of the $\mathrm{ER} \alpha$ target gene expression.

ERAP suppresses E2-dependent non-genomic ER $\alpha$ signalling. In addition to $\mathrm{ER} \alpha$ acting as a nuclear transcription factor, E2 rapidly induces IGF-1R $\beta$ tyrosine phosphorylation followed by the formation of a ternary complex of IGF-1R $\beta, E R \alpha$ and Shc in the cell membrane ${ }^{9}$, even though the abundance of membranebound and cytoplasmic ER $\alpha$ is low in primary breast cancers ${ }^{34}$. Indeed, we observed that a portion of PHB2 released from BIG3 by ERAP interacted with $\mathrm{ER} \alpha$ in the cytoplasmic/plasma membrane cell fraction, regardless of the presence of E2 (Fig. 3a,b and Supplementary Fig. S3a,b). Therefore, we hypothesized that ERAP could also affect these non-genomic actions of ER $\alpha$. First, we detected E2-induced tyrosine phosphorylation of IGF-1R $\beta$ and co-immunoprecipitated IGF-1R $\beta, E R \alpha$ and Shc in both MCF-7 and KLP-3C cells (Fig. 4a), which highly expressed IGF$1 R \beta$ and PI3K (Supplementary Fig. S4a), as described previously ${ }^{9}$. In contrast, ERAP treatment removed Shc from this complex and formed a new ternary complex consisting of IGF-1R $\beta, \mathrm{ER} \alpha$ and $\mathrm{PHB} 2$, and thereby suppressed E2-induced tyrosine phosphorylation of IGF-1R $\beta$ (Fig. 4a). We then examined the effects of ERAP on the phosphorylation of membrane-associated
$\mathrm{ER} \alpha$ (S118), because its phosphorylation has been associated with invasive breast cancer in clinical specimens ${ }^{35}$. ERAP treatment clearly suppressed the E2-induced phosphorylation (S118) of membrane-associated $\mathrm{ER} \alpha$ in the IGF-1R $\beta$-precipitated membrane fraction of MCF-7 cells (Supplementary Fig. S4b). Moreover, ERAP also interfered with the E2-induced interactions of $\mathrm{ER} \alpha$ and PI3K in both MCF-7 and KLP-3C cells (Fig. 4b). Next, we examined the effects of ERAP on the phosphorylation status of Akt and p42/44 MAPK, which are the downstream signalling molecules of IGF-1R $\beta$ and PI3K, respectively. As expected, we observed that Akt (S473) and p42/44 MAPK (T202/Y204) phosphorylation levels were clearly increased in a time-dependent manner after E2 stimulation in both cell lines, whereas treatment with ERAP, but not scrERAP, completely suppressed the E2-induced phosphorylation levels of both proteins (Fig. 4c and Supplementary Fig. S4c). However, the PHB2 released from BIG3 following ERAP treatment did not directly interact with Akt or p42/44 MAPK (Supplementary Fig. S4d). Taken together, these results strongly suggested that ERAP interfered with E2-induced non-genomic ER $\alpha$ activation pathways, such as those mediated by IGF-1R $\beta$.

ERAP represses E2-dependent ER $\alpha$ phosphorylation. Accumulating evidence suggests that phosphorylation of $E R \alpha$ is an important regulator of E2-induced ER $\alpha$ transcriptional activity, DNA-binding, co-activator binding, and protein stability and cell proliferation in ER $\alpha$-positive breast cancer cells $s^{36-43}$. Thus, we examined the effects of ERAP on ER $\alpha$ phosphorylation at sites, including S104/S106, S118, S167, S305 and Y537. 
a
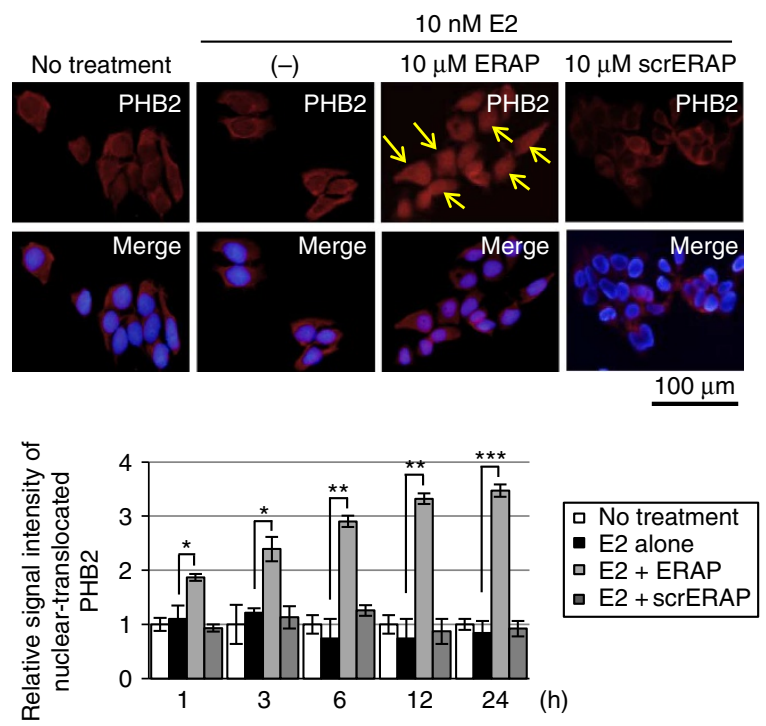

b

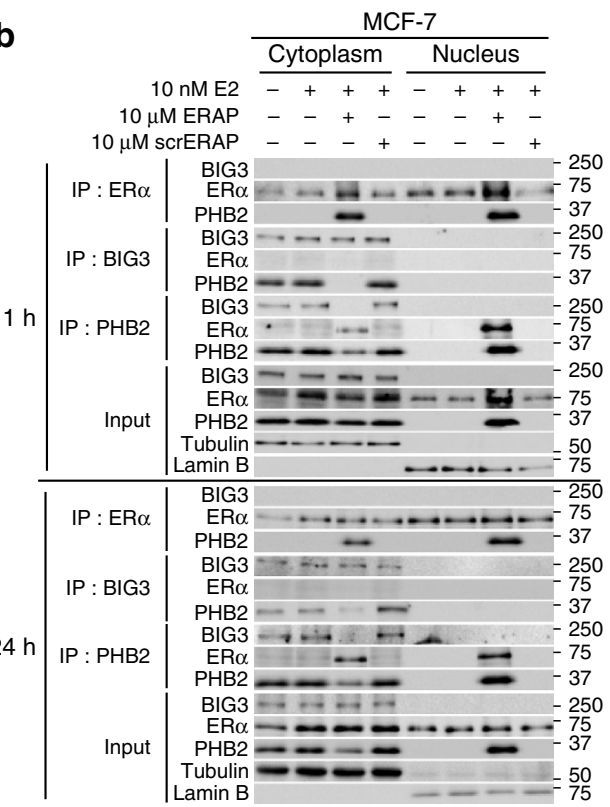

C

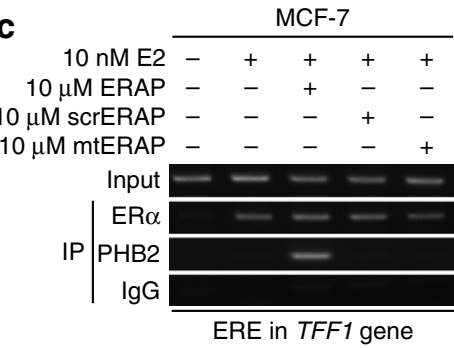

d

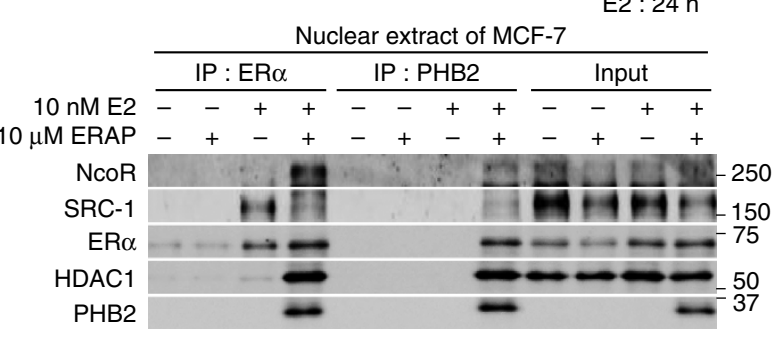

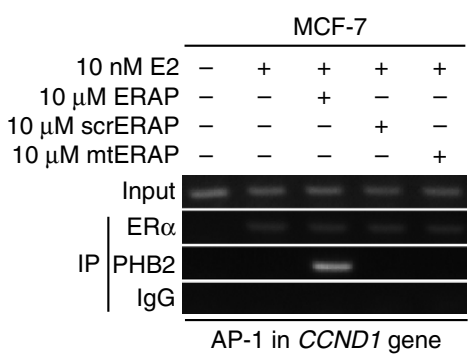

e

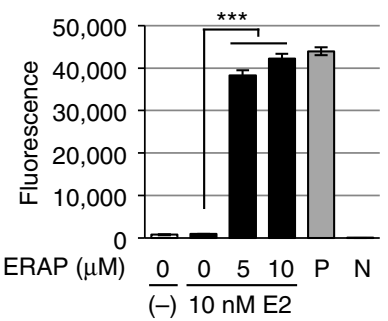

f

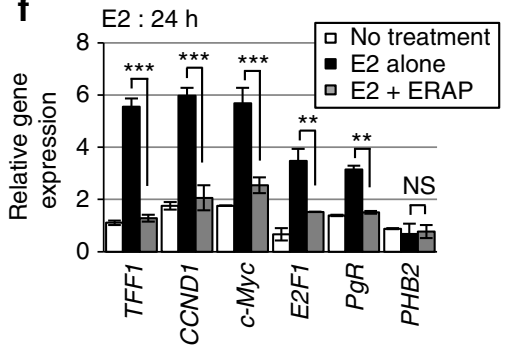

Figure 3 | ERAP promotes PHB2 nuclear translocation and suppresses E2-induced gene expression. (a) Upper, representative immunofluorescence images of the subcellular localization of PHB2 are shown; PHB2 (red), 4',6-diamidino-2-phenylindole (blue). The arrows indicate PHB2 nuclear translocation. Lower, statistical analyses of the nuclear intensity of translocated PHB2. The data are expressed as the fold increase over untreated cells set at 1.0 and represent the mean \pm s.e.m. of four independent experiments $\left({ }^{\star} P<0.05,{ }^{\star \star} P<0.01,{ }^{\star \star \star} P<0.001\right.$ in two-sided Student's $t$-test). (b) Immunoblot analysis was performed to detect the subcellular localization of $E R \alpha, B I G 3$ and PHB2 after immunoprecipitation with the antibodies against the indicated proteins in the presence of E2 and ERAP or scrERAP. $\alpha / \beta$-Tubulin (tubulin) and laminin B were used as loading controls for the cytoplasmic and nuclear fractions, respectively. (c) ChIP assays were used to determine the recruitment of ER $\alpha$ and PHB2 to the TFF1 ERE sequence (left) and the CCND1 AP-1 motif (right) after $24 \mathrm{~h}$ of treatment. (d) Exchange of chromatin-remodelling complexes by ERAP. After treatment of MCF-7 cells for $24 \mathrm{~h}$ with E2 \pm ERAP, the nuclear fractions were immunoprecipitated with anti-ER $\alpha$ or -PHB2 antibodies and were immunoblotted with antibodies against the indicated proteins. (e) Deacetylation of chromatin-remodelling complexes was evaluated after ERAP treatment. Nuclear extracts from HeLa cells and PBS were used as positive $(P)$ and negative $(N)$ controls, respectively. The data represent the mean \pm s.e.m. of three independent experiments ( ${ }^{\star \star \star} P<0.001$ in two-sided Student's $t$-test). (f) The effects of ERAP on ER $\alpha$-target gene expression levels were evaluated using real-time PCR. The data are expressed the fold increase over untreated cells at $\mathrm{O}$ h (set at 1.0) and represent the mean \pm s.e.m. of three independent experiments $\left({ }^{\star \star} P<0.01,{ }^{\star \star \star} P<0.001, \mathrm{NS}\right.$, no significance in two-sided Student's $t$-test).

Phosphorylation at these five sites within ER $\alpha$ was clearly increased in response to E2 stimulation and continued for at least $24 \mathrm{~h}$ in MCF-7 and KPL-3C cells. In contrast, treatment with ERAP completely abrogated these responses in both cell lines (Fig. 4d and Supplementary Fig. S4e). Collectively, these results clearly showed that PHB2 released from BIG3 following ERAP treatment reduced $\mathrm{E} 2$-dependent $\mathrm{ER} \alpha$ phosphorylation, leading to $\mathrm{ER} \alpha$ inactivation.
ERAP suppresses E2-dependent breast cancer cell growth. We elucidated the inhibitory effect of ERAP on the E2-dependent growth of MCF-7 or KPL-3C cells. Treatment with ERAP, but not scrERAP or mtERAP, significantly reduced E2-stimulated cell growth in a dose-dependent manner $\left(\mathrm{IC}_{50}=2.2 \mu \mathrm{M}\right.$ and $1.9 \mu \mathrm{M}$ in MCF-7 and KPL-3C cells, respectively; Fig. 5a). Notably, ERAP doses greater than $5 \mu \mathrm{M}$ completely abolished the proliferative response for up to $3 \mathrm{~h}$ after E2 stimulation 
a

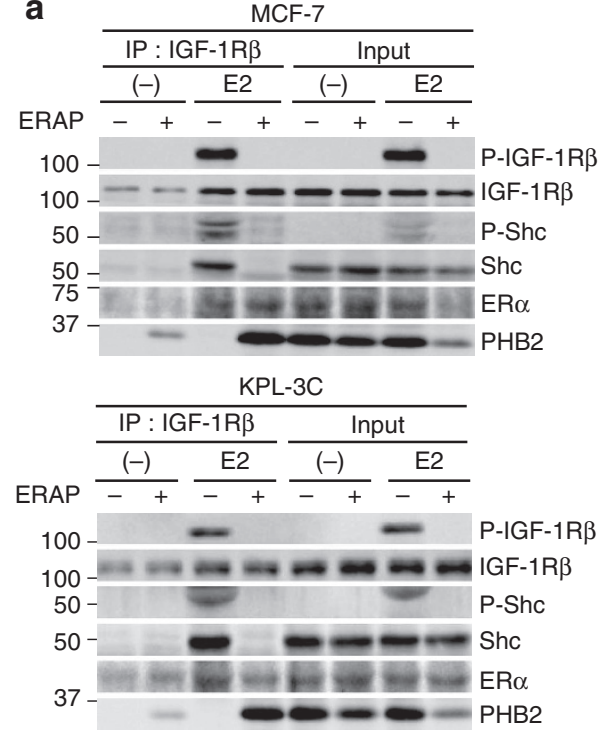

b

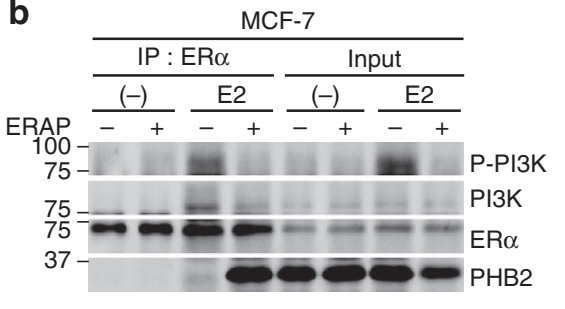

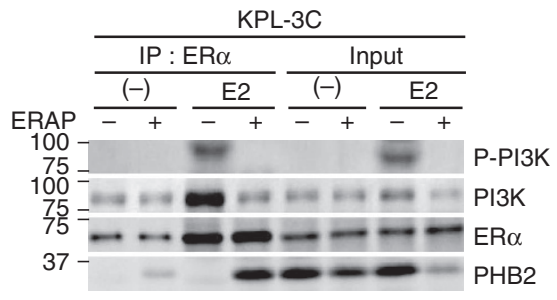

C

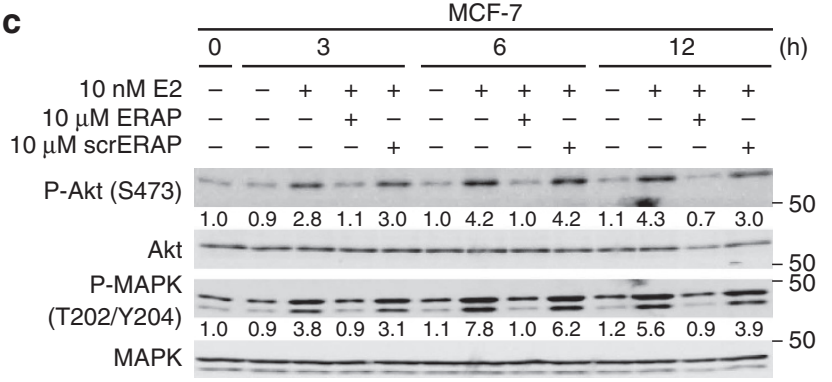

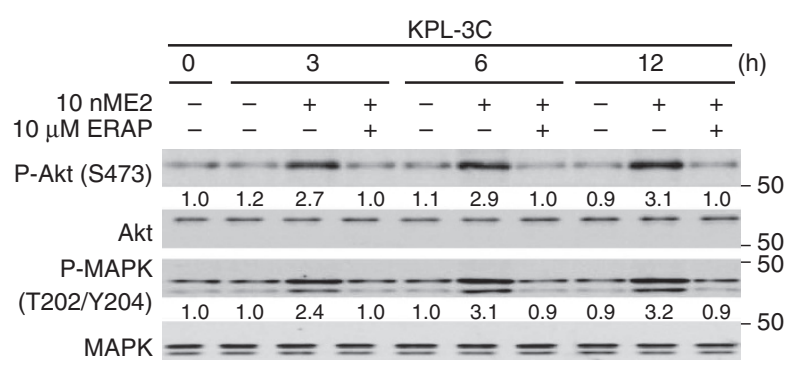

d

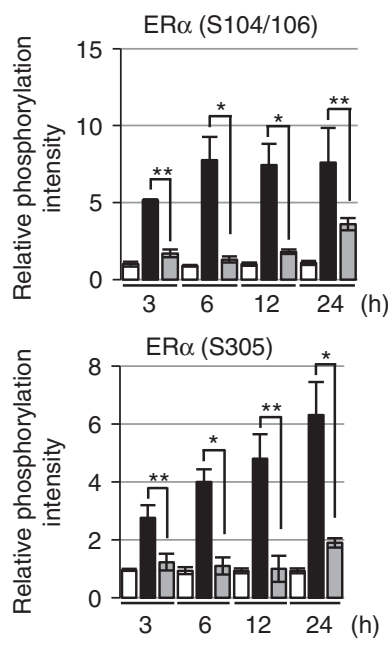

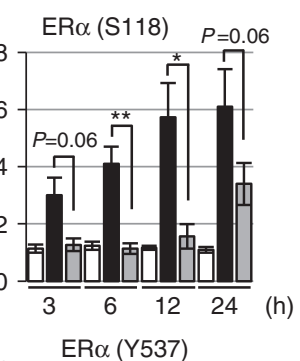

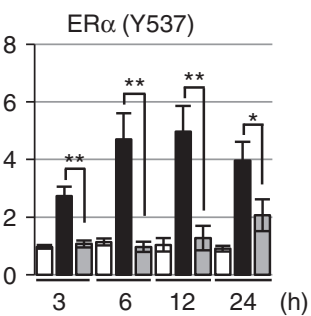

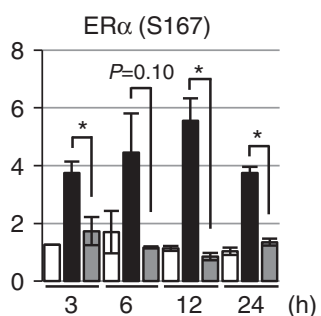

(h)

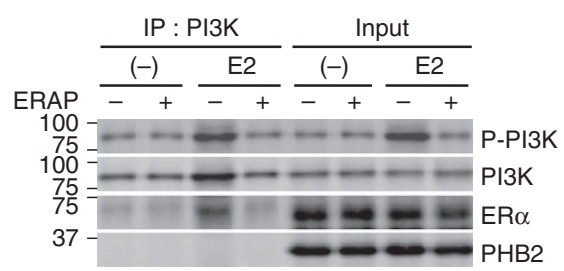

Figure 4 | ERAP regulates the E2-induced non-genomic pathway via IGF-1Rß. (a,b) The inhibitory effects of ERAP on the interactions of ER $\alpha$ with IGF-1R $\beta$ and Shc (a), and the interaction of ER $\alpha$ and PI3K (b) in MCF-7 and KPL-3C cells. (c) Immunoblot analyses were performed to evaluate the inhibitory effects of ERAP on E2-induced Akt (S473) and p42/44 MAPK (T202/Y204) activities in MCF-7 (left) and KPL-3C (right) cells. Representative results are shown from one of four independent experiments. (d) The inhibitory effects of ERAP were evaluated on E2-induced phosphorylation of ER $\alpha$ (S104/S106, S118, S167, S305 and Y537) in MCF-7 cells. The data are expressed as the fold increase over untreated cells at $0 \mathrm{~h}$ and represent the mean \pm s.e.m. of three independent experiments $\left({ }^{\star} P<0.05,{ }^{\star \star} P<0.01\right.$ in two-sided Student's $t$-test).

(Supplementary Fig. S5a). The inhibition of both cell growth (Supplementary Fig. S5b, left panel) and ER $\alpha$ transcriptional activity (Supplementary Fig. S5b, right panel) was maintained for $24 \mathrm{~h}$ after ERAP treatment. We confirmed similar growth inhibitory effects of ERAP in other breast cancer cell lines expressing ER $\alpha$, BIG3 and PHB2 (that is, ZR-75-1, HCC1500,
BT-474, YMB-1, T47D, KPL-1 and HBC4; Supplementary Fig. S5c). In contrast, ERAP had no effect on the growth of normal mammary epithelial MCF-10 A cells (Fig. 5b) that did not express $\mathrm{ER} \alpha$ or BIG3 (Supplementary Fig. S2). These findings suggested that ERAP specifically inhibited the growth of breast cancer cells without affecting normal mammary cells. 
a

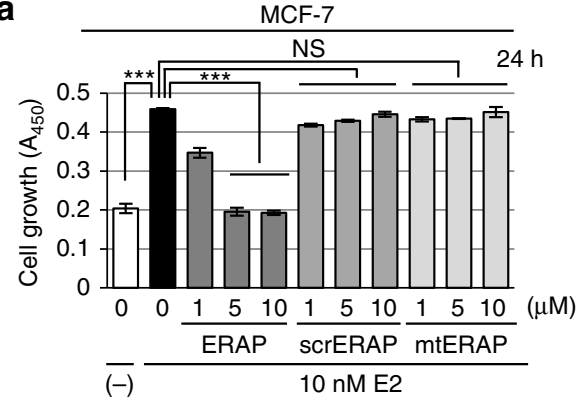

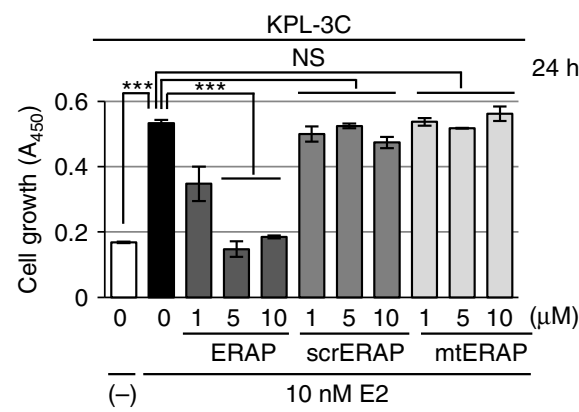

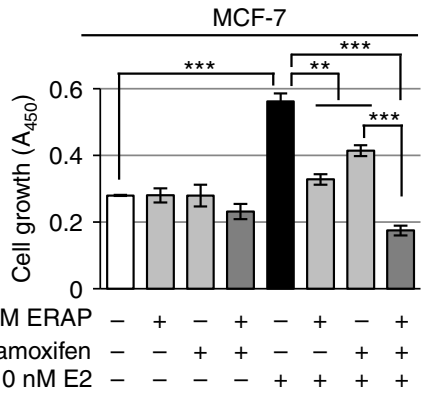

d

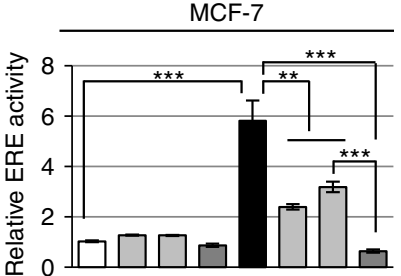

$10 \mu \mathrm{M}$ ERAP $-+\ldots+++$ $10 \mathrm{nM}$ tamoxifen $-\quad++-++$ $10 \mathrm{nME2}-\ldots+\ldots++$

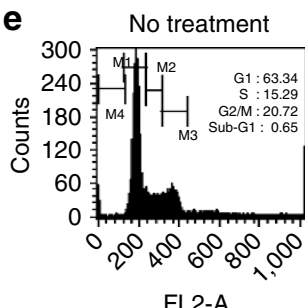

FL2-A

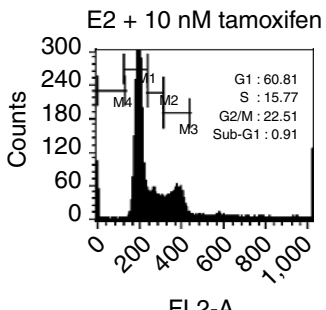

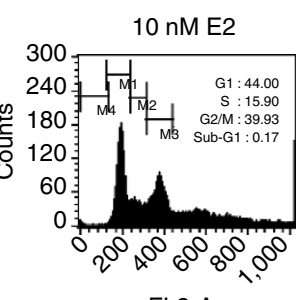

FL2-A

E2 + 10 mM ERAP

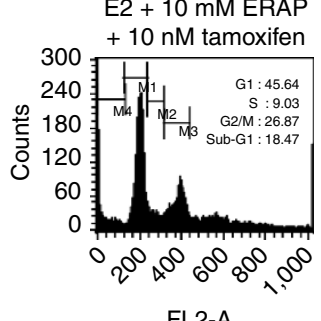

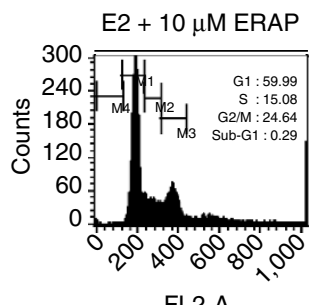

FL2-A

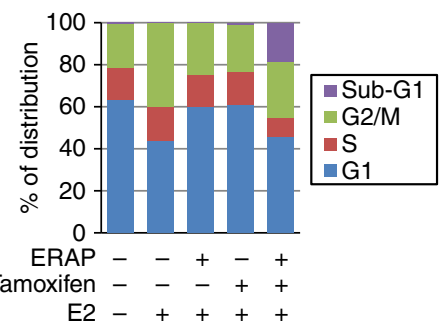

Figure 5 | ERAP suppresses growth of ER $\boldsymbol{\alpha}$-dependent breast cancer cell lines in vitro. (a,b) An MTT (3-(4,5-dimethylthiazol-2-yl)-2,5-

diphenyltetrazolium bromide) assay was performed to evaluate the inhibitory effect of ERAP on E2-dependent growth of the BIG3-positive MCF-7 and KPL-3C (a), and the BIG3-negative mammary epithelial cell line MCF-10A (b). The data represent the mean \pm s.e.m. of three independent experiments ${ }^{\star \star \star} P<0.001$ in two-sided Student's $t$-test). (c,d) The combined inhibitory actions of ERAP and tamoxifen were evaluated using MTT assays (c) and luciferase assays (d). MCF-7 cells were treated for $24 \mathrm{~h}$ with E2 \pm ERAP, tamoxifen, or a combination of ERAP and tamoxifen. The data of all panels represent the mean \pm s.e.m. of three independent experiments ${ }^{\star \star} P<0.01,{ }^{\star \star \star} P<0.001$ in two-sided Student's $t$-test). (e) Flow cytometric analyses were performed to evaluate the effect of ERAP treatment on the cell cycle. MCF-7 cells were treated for $24 \mathrm{~h}$ with E2 \pm ERAP, tamoxifen, or a combination of ERAP and tamoxifen.

Furthermore, treatment with a combination of ERAP and tamoxifen significantly suppressed E2-induced cell growth (Fig. 5c) and ER $\alpha$ transcriptional activity (Fig. 5d) in MCF-7 cells as compared with ERAP or tamoxifen alone. Next, we examined the effects of ERAP on the cell cycle distribution of MCF-7 cells using flow cytometry. The population of cells in the G2/M phase increased after a $24 \mathrm{~h}$ E2 stimulation, whereas the population in the G1 phase increased after ERAP or tamoxifen treatment, suggesting that ERAP suppressed cell growth by inducing a G1 arrest, similar to tamoxifen ${ }^{44}$ (Fig. 5e). Importantly, a remarkable increase in the apoptotic (sub-G1) cell population was observed after treatment with a combination of
ERAP and tamoxifen (18.47\%), although no phenotypic alterations or increases in the sub-G1 population were observed after treatment with ERAP, tamoxifen or E2 alone $(0.29 \%, 0.91 \%$ or $0.17 \%$, respectively; Fig. 5 e). Taken together, our data strongly suggest that ERAP enhanced the responsiveness of ER $\alpha$-positive breast cancer cells to tamoxifen.

Anti-tumour efficacy of ERAP in xenograft models. To determine whether ERAP could affect the growth of ER $\alpha$-positive breast cancer tumours in vivo, we developed KPL-3C (Fig. 6a) and MCF-7 (Supplementary Fig. S6a) orthotopic breast cancer 
a

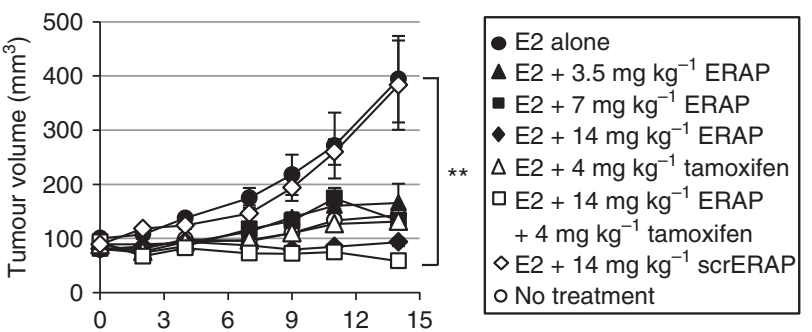

b

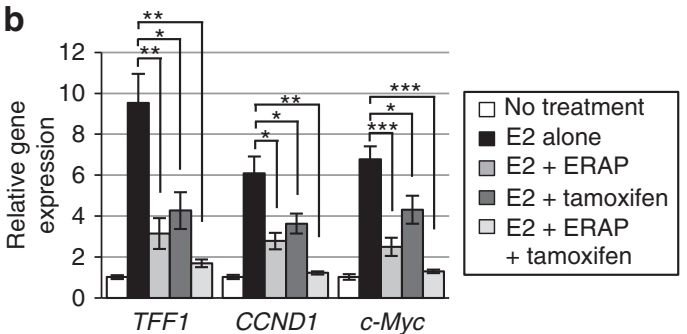

C

Tumour of KPL-3C xenograft

$(-1)$ $6 \mu \mathrm{g}$ per day E2

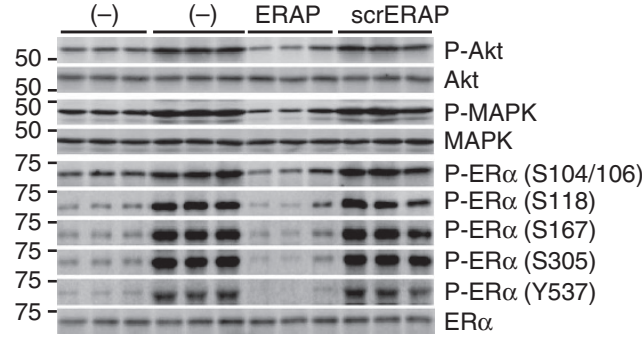

Day 15 ERo

Figure 6 | ERAP inhibits tumour growth in xenograft models of human ER $\alpha$-positive breast cancer. (a) ERAP inhibits tumour growth in a human breast cancer KPL-3C xenograft mouse model. The tumour volume represents the mean \pm s.e.m. of each group $\left(n=5 ;{ }^{\star \star} P<0.01\right.$ in two-sided Student's t-test). (b) Real-time PCR was performed to determine the combined inhibitory effects of ERAP and tamoxifen on the expression of ER $\alpha$ target genes. The data are expressed the fold increase over untreated cells at $0 \mathrm{~h}$ (set at 1.0) and represent the mean \pm s.e.m. of each group $\left(n=5 ;{ }^{\star} P<0.05,{ }^{\star \star} P<0.01\right.$, ${ }^{\star \star \star} P<0.001$ in two-sided Student's $t$-test). (c) Immunoblot analyses were performed to evaluate the effects of ERAP on the phosphorylation levels of Akt, $\mathrm{p} 42 / 44$ MAPK and $E R \alpha$ proteins in tumours.

xenografts in nude mice. Daily treatment with E2 alone induced time-dependent growth of KPL-3C and MCF-7 tumours, whereas treatment with $14 \mathrm{mg} \mathrm{kg}^{-1}$ ERAP caused significant inhibition of E2-induced tumour growth compared with mice treated with E2 alone or scrERAP in both tumour cell lines $\left(14 \mathrm{mg} \mathrm{kg}^{-1} ; n=5\right.$; $P<0.01$ in two-sided Student's $t$-test; Fig. $6 \mathrm{a}$ and Supplementary Fig. S6a). No toxicity or significant body weight changes were observed in either xenograft throughout these experiments (Supplementary Fig. S6b). As expected, a significant reduction in mRNA levels was evident for TFF1, CCND1, and $c-M y c$ in tumours treated with ERAP compared with those treated with scrERAP or vehicle only (Fig. $6 \mathrm{~b}$ and Supplementary Fig. S6c). Furthermore, considerable suppression of Akt, p42/44 MAPK and $E R \alpha$ phosphorylation was observed in tumours treated with ERAP (Fig. 6c and Supplementary Fig. S6d). More importantly, the combined treatment of ERAP and tamoxifen additively inhibited the expression of $\mathrm{ER} \alpha$ target genes (Fig. 6b) and the development of KPL-3C xenografts as compared with tamoxifen or ERAP alone (Fig. 6a). These results demonstrated that ERAP had in vivo anti-tumour activity and could enhance the antitumour effects of tamoxifen.

ERAP suppresses growth of TAM-R tumours. To confirm that ERAP had an anti-tumour effect against endocrine-resistant breast cancer, we first examined the effects of ERAP on the activation of the non-genomic signalling pathway, on the phosphorylation of $E R \alpha$ and on the non-canonical ER $\alpha$ transcriptional activation via AP-1, which is responsible for tamoxifen resistance $^{10,11,16}$ in TAM-R MCF-7 (ref. 45) and T47D (ref. 46) cells. The phosphorylation levels of Akt, p42/44 MAPK and $\mathrm{ER} \alpha$ were enhanced in response to tamoxifen alone or a combination of tamoxifen and E2, whereas ERAP treatment clearly suppressed these responses in both cell types (Fig. 7a,b and Supplementary Fig. S7a). ERAP treatment also clearly suppressed the phosphorylation levels of Akt, p42/44 MAPK and ER $\alpha$ in response to a combination of E2 and IGF-2 in the presence of tamoxifen in TAM-R T47D cells (Fig. 7b). In addition, ERAP significantly inhibited E2-induced ER $\alpha$ transcriptional activity via AP-1, as well as ERE (Supplementary Fig. S7b), and the E2-induced expression of TFF1, CCND1 and $c-M y c$ genes (Supplementary Fig. S7c,d) in the presence of tamoxifen in TAM-R MCF-7 or TAM-R T47D cells.

Next, we tested the ability of ERAP to inhibit the E2-dependent growth of TAM-R cells and found that ERAP treatment significantly reduced the growth of TAM-R MCF-7 (Fig. 7c) and TAM-R T47D cells (Fig. 7d) in the presence of tamoxifen. Furthermore, we examined the inhibitory effects of ERAP on the E2-dependent tumour growth of TAM-R MCF-7 and T47D orthotropic breast cancer xenografts in nude mice. The results demonstrated that E2-induced growth of both TAM-R tumours was suppressed by treatment with $14 \mathrm{mg} \mathrm{kg}^{-1} \operatorname{ERAP}(n=4$; $P<0.001$, Fig. 7e; $n=5 ; P<0.01$ in two-sided Student's $t$-test, Fig. 7f). Furthermore, considerable suppression of Akt, p42/44 MAPK and ER $\alpha$ phosphorylation was observed in both TAM-R tumours treated with ERAP (Supplementary Fig. S7e,f). Collectively, these data suggested that ERAP acted as an effective therapeutic agent with respect to endocrine-resistant breast cancer.

E2-dependent direct transactivation of BIG3 by ER $\alpha$. It was previously reported that $B I G 3$ is upregulated after E2 treatment in MCF-7 cells ${ }^{21}$. Thus, we hypothesized that BIG3 may be a potential target gene of $\mathrm{ER} \alpha$ and found that its expression was significantly upregulated in MCF-7 cells in a time-dependent manner after E2 stimulation (Fig. 8a). Interestingly, we also noted a significant reduction in BIG3 expression at both the transcriptional and protein levels in a dose-dependent manner after tamoxifen treatment (Fig. 8b). Accordingly, to obtain direct 
a

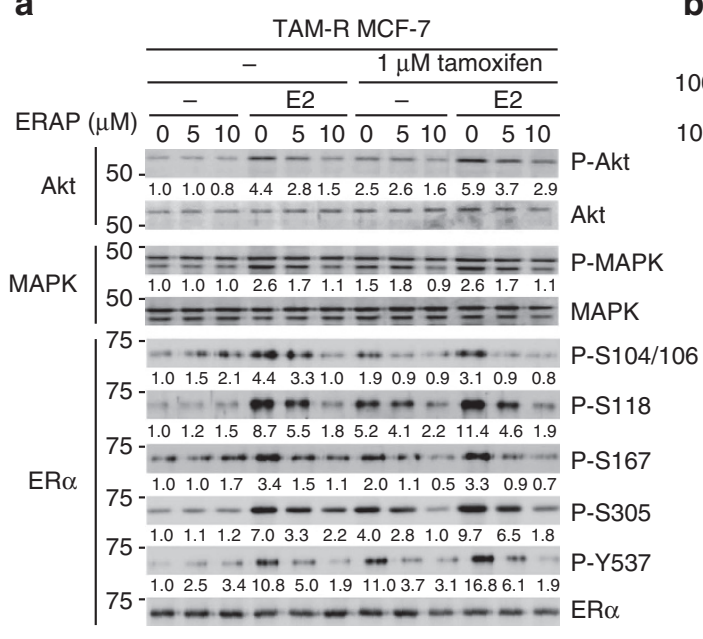

C

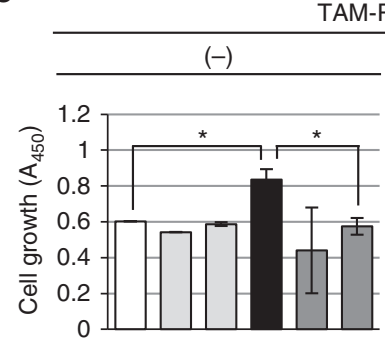

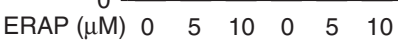

10 nME2 - $\quad+\quad++$

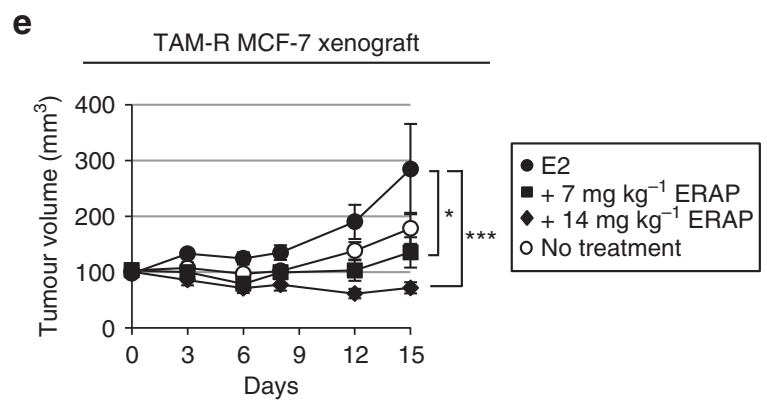

b

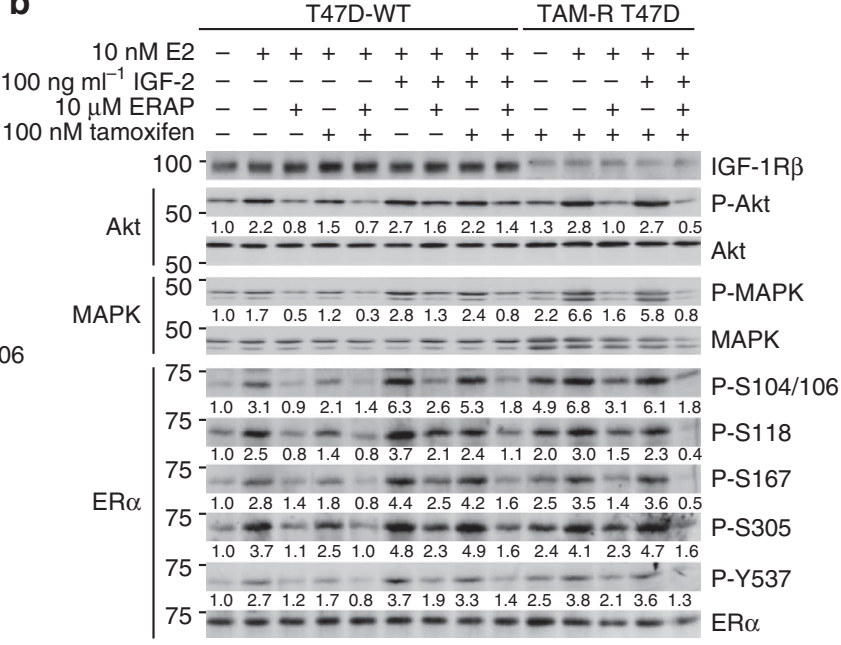

d

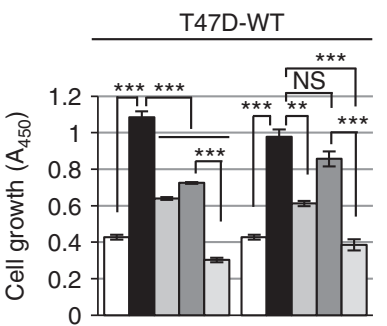

$10 \mu$ M ERAP --+-+--+-+ $10 \mathrm{nM}$ tamoxifen ---++---++

$10 \mathrm{nME} 2-++++-++++$

$100 \mathrm{ng} \mathrm{ml}^{-1}$ IGF-2 $-\ldots-\ldots++++$

TAM-R T47D

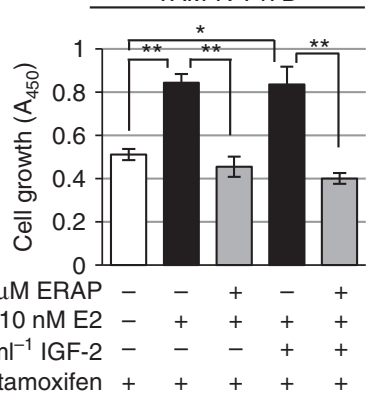

f
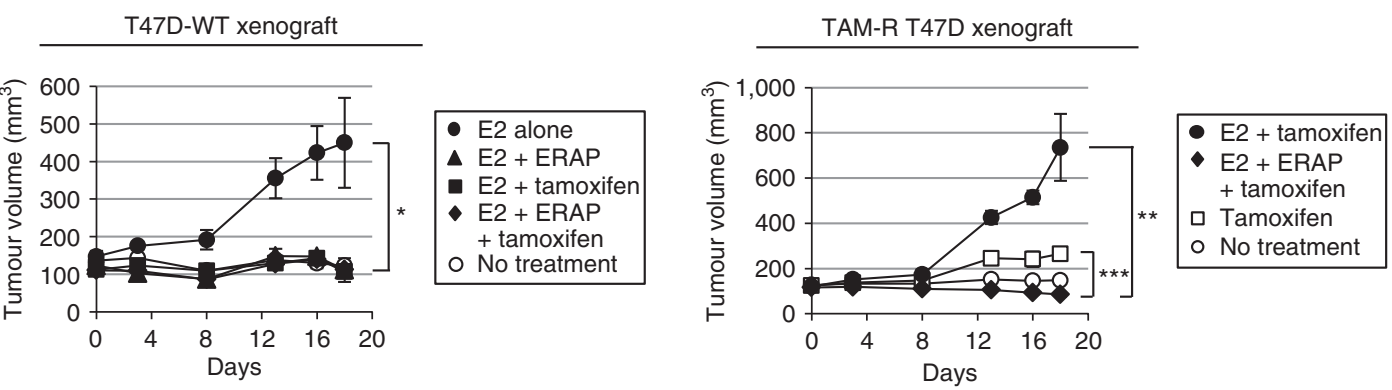

Figure 7 | ERAP suppresses growth of TAM-R tumours. (a,b) Immunoblot analyses were performed to evaluate the effects of ERAP on the phosphorylation levels of Akt, p42/44 MAPK and ER $\alpha$ proteins in TAM-R MCF-7 (a), and a parent T47D (T47D-WT) and TAM-R T47D (b) cells. (c) MTT (3-(4,5dimethylthiazol-2-yl)-2,5-diphenyltetrazolium bromide) assays were performed to evaluate the inhibitory effect of ERAP on the growth of TAM-R MCF-7 cells. TAM-R MCF-7 cells were treated for $24 \mathrm{~h}$ with E2 \pm ERAP in the presence or absence of $1 \mu \mathrm{M}$ tamoxifen. The data represent the mean \pm s.e.m. of three independent experiments ( ${ }^{\star} P<0.05,{ }^{\star \star \star} P<0.001$ in two-sided Student's $t$-test). (d) Inhibitory effect of ERAP on the growth of T47D-WT (upper) and TAM-R T47D (lower) cells after $24 \mathrm{~h}$ of treatment with E2 alone or E2 and IGF-2 in the presence and the absence of $10 \mathrm{nM}$ tamoxifen, respectively. The data of all panels represent the mean \pm s.e.m. of three independent experiments $\left({ }^{\star} P<0.05,{ }^{\star \star} P<0.01,{ }^{\star \star \star}{ }^{\star} P<0.001\right.$ in two-sided Student's $t$-test). (e) ERAP inhibits the tumour growth of TAM-R MCF-7 orthotropic breast cancer xenografts in nude mice. The tumour volume represents the mean \pm s.e.m. of each group $(n=5)\left({ }^{\star} P<0.05\right.$, ${ }^{\star \star \star} P<0.001$ in two-sided Student's $t$-test). (f) ERAP inhibits the tumour growth of both T47D-WT (left) and TAM-R T47D (right) orthotropic breast cancer xenografts in nude mice. The tumour volume represents the mean \pm s.e.m. of each group $\left(n=5 ;{ }^{\star} P<0.05,{ }^{\star \star} P<0.01\right.$, ${ }^{\star \star \star} P<0.001$ in two-sided Student's $t$-test). 
a

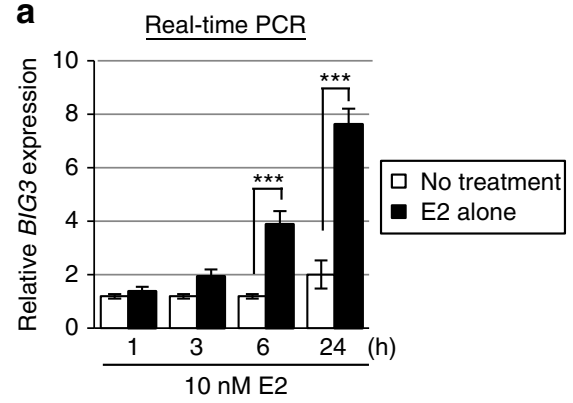

C

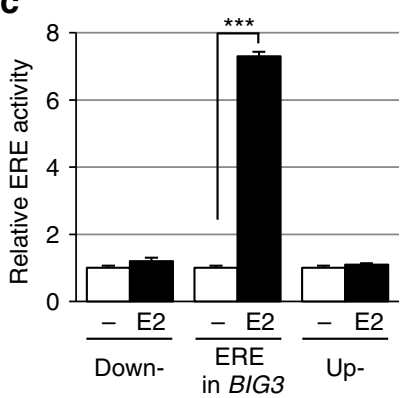

ERE consensus in intron1 of BIG3 gene

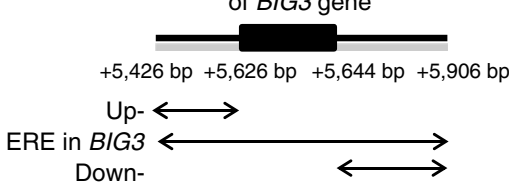

b
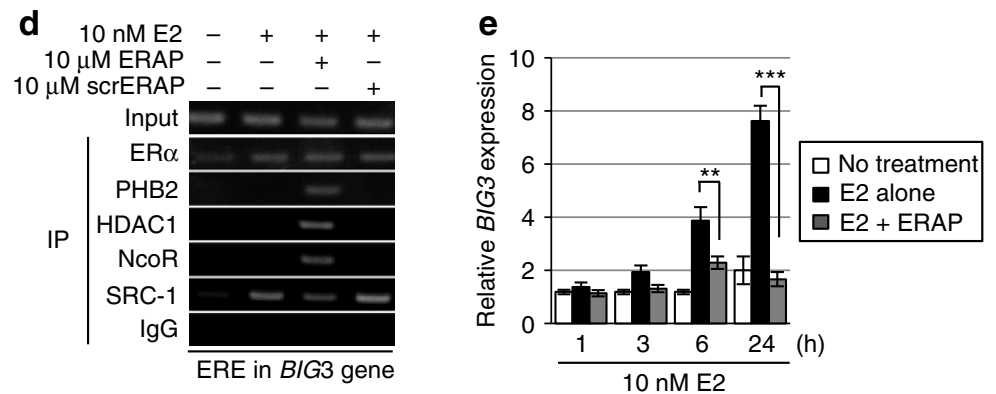

f

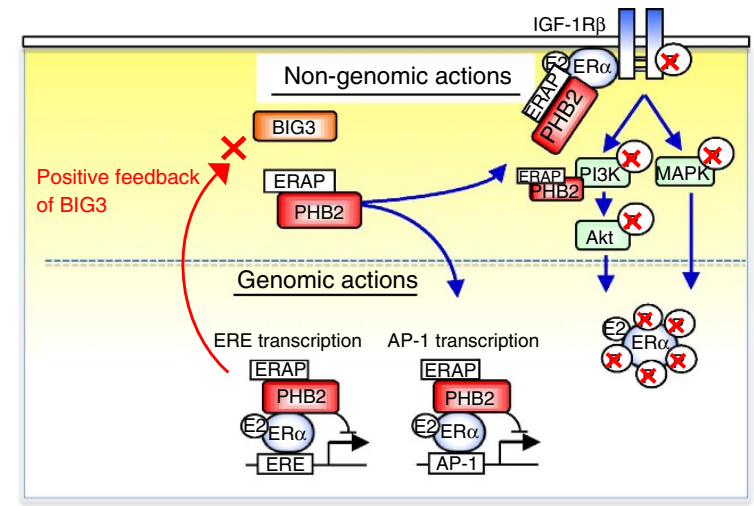

Figure 8 | Positive feedback regulation of BIG3 transactivation. (a) Upregulation of BIG3 expression was evaluated after E2 stimulation. These data are expressed the fold increase over untreated cells at $\mathrm{O}$ h (set at 1.0) and represent the mean \pm s.e.m. of three independent experiments ( ${ }^{\star \star \star} P<0.001$ in twosided Student's $t$-test). (b) The effects of tamoxifen were evaluated on BIG3 expression. For real-time PCR analyses (left), the data are expressed as the fold increase over untreated cells (set at 1.0). These data represent the mean \pm s.e.m. of three independent experiments ${ }^{\star \star} P<0.01$, ${ }^{\star \star \star} P<0.001$ in two-sided Student's t-test). For immunoblot analyses (right), $\beta$-actin served as a loading control. (c) Luciferase assays were performed to evaluate the transactivation of BIG3 using a luciferase reporter containing an ERE motif conserved within intron 1 of the BIG3 gene (5'-TCCAGTTGCATTGACCTGA-3'; 5,626-5,644 bp from the transcriptional start site of $B I G 3$ ) or constructs containing the upstream and downstream regions lacking this ERE motif. The data represent the mean \pm s.e.m. of three independent experiments ( ${ }^{\star \star} P<0.001$ in two-sided Student's $t$-test). (d) ChIP assays show the transactivation of $B I G 3$ through an intronic ERE. (e) The effect of ERAP on BIG3 expression using real-time PCR is shown at the indicated time points. The data are expressed the fold increase over untreated cells at $\mathrm{O}$ (set at 1.0) and represent the mean \pm s.e.m. of three independent experiments ( ${ }^{\star \star} P<0.01,{ }^{\star \star \star} P<0.001$ in two-sided Student's $t$-test). (f) ERAP treatment leads to multiple levels of inhibition targeted at E2-dependent ER $\alpha$ activation pathways. ERAP competitively binds to endogenous PHB2, thereby preventing its interactions with BIG3. Free PHB2 directly binds to both nuclear and membrane-associated ER $\alpha$, resulting in the repression of E2-induced ER $\alpha$ activation through non-genomic pathways and its phosphorylation. Moreover, ERAP also attenuates BIG3 transcription, resulting in the downregulation of ER $\alpha$ target genes, including $B I G 3$.

evidence for the upregulation of BIG3 expression by $\mathrm{ER} \alpha$, we measured $E R \alpha$ transcriptional activity. E2 stimulation resulted in robust luciferase activity only in cells transfected with the construct containing an intronic ERE from BIG3 (Fig. 8c), suggesting potential transactivation of $B I G 3$ by ER $\alpha$. Next, we examined the recruitment of ER $\alpha$ to an intronic ERE motif of the BIG3 gene by ChIP analysis (Fig. 8d). E2-dependent recruitment of ER $\alpha$ and the co-activator SRC-1 was observed associated with an ERE within intron 1 of the BIG3 gene in MCF-7 cells. In contrast, ERAP treatment enhanced recruitment of HDAC1 and NcoR to the ER $\alpha-\mathrm{PHB} 2$ complex (Fig. 8d). In addition, ERAP treatment led to significant suppression of the E2-induced expression levels of $B I G 3$ even after $3 \mathrm{~h}$ (Fig. $8 \mathrm{e}$ ). These results demonstrated that
$B I G 3$ was directly transactivated by $\mathrm{ER} \alpha$ via its intronic ERE following E2 treatment, which suggested that BIG3 acts through a positive feedback mechanism to enhance ER $\alpha$ activation in E2dependent breast cancer cells. In other words, ERAP blocked this positive regulation of $B I G 3$, leading to the release of PHB2 from cytoplasmic BIG3 and the inhibition of $\mathrm{ER} \alpha$ activity via multiple mechanisms.

\section{Discussion}

In this study, we developed a dominant-negative peptide, ERAP, based on the residues Q165, D169 and Q173 in BIG3, which were essential for its heterodimerization with $\mathrm{PHB} 2$ to 
suppress the growth of $E R \alpha$-positive breast cancer cells, especially endocrine-resistant breast cancer cells. ERAP competitively bound endogenous PHB2, thereby preventing its interaction with BIG3 and releasing PHB2 to directly bind to both nuclear- and membrane-associated $\mathrm{ER} \alpha$. Intrinsic PHB2 binding to $\mathrm{ER} \alpha$ led to the repression of a number of E2-induced activation events, which resulted in complete suppression of E2-dependent ER $\alpha$ positive breast cancer cell growth in vitro and in vivo (Fig. 8f). These findings suggest that PHB2 has an important role in the modulation of multiple aspects of the E2/ER $\alpha$-signalling network, although PHB2 has been reported to be the only transcriptional repressor for $\mathrm{ER} \alpha$. However, to date, it remains unclear how the endogenous tumour suppressor PHB2 is inactivated in cancer cells, despite abundant expression and the lack of genomic mutations or methylations in breast cancer clinical specimens or cell lines ${ }^{47}$. Our data suggest a potential solution to this unanswered problem; namely, our results suggest that BIG3 may sequester PHB2 in cancer cells, thereby causing an apparent 'lossof-function' of PHB2 protein. These findings mark the first step toward uncovering a new E2/ER $\alpha$ signalling network in breast cancer and shed light on novel therapeutic strategies using PHB2 protein functions for $\mathrm{E} 2 / \mathrm{ER} \alpha$-positive breast cancer.

The most important finding of this study was that ERAP might potentially act as an effective inhibitor of TAM-R breast cancer cells. Current endocrine therapies for breast cancer are based mainly on targeting the ER $\alpha$-signalling pathway, and tamoxifen is the most frequently prescribed drug for the treatment of all stages of breast cancer ${ }^{12,13}$. However, tamoxifen resistance is a major clinical problem and a leading cause of treatment failure and mortality ${ }^{14,15}$. Compelling evidence suggests that multiply phosphorylated $\mathrm{ER} \alpha$ and non-canonical ER $\alpha$ activation via AP1 are linked to tamoxifen or aromatase inhibitor resistance in breast cancer cells ${ }^{10,11,16}$. In addition, the activation of the IGF1Rß/AKT-ERK1/2-MAPK network is also linked to tamoxifen resistance in breast cancers ${ }^{10,11,16}$, even though the abundance of membrane-bound and cytoplasmic ER $\alpha$ is low in primary breast cancers ${ }^{34}$. Our study demonstrated that ERAP treatment completely inhibited ER $\alpha$-IGF-1R $\beta$ and/or ER $\alpha$-PI3K interactions, ER $\alpha$ phosphorylation at multiple sites and ER $\alpha$ transcriptional activation via $\mathrm{AP}-1$ in the presence of $\mathrm{E} 2$ in $E R \alpha$-positive breast cancer cells. Indeed, ERAP had significant anti-tumour effects against TAM-R breast cancer cells. More importantly, we revealed that the combination of tamoxifen and ERAP induced rapid apoptosis and exhibited more potent antitumour activity in vivo and in vitro as compared with either treatment alone, indicating enhanced tamoxifen responsiveness. This combined effect is thought to be due to the distinct mechanisms of action of each drug, suggesting that releasing intrinsic PHB2 could suppress multifactorial mechanisms of endocrine resistance.

Current endocrine therapies, such as tamoxifen, pinpoint specific signalling pathways or molecules in the ER $\alpha$-signalling network. In contrast, ERAP was shown to modulate multiple aspects of the E2/ER $\alpha$-signalling network via the tumour suppressive ability of endogenous PHB2, which was expressed abundantly in cancer cells, leading to the complete suppression of E2-dependent breast cancer cell growth. Moreover, although $B I G 3$ is transactivated directly by ER $\alpha$ in a positive feedback loop, ERAP was shown to downregulate BIG3 transcription, which reduced BIG3 protein levels in the cytoplasm of cancer cells and enabled endogenous PHB2 to suppress the extensive ER $\alpha$ signalling network. Moreover, as BIG3 is specifically upregulated in breast cancer but is hardly detectable in normal human tissues, agents such as ERAP, which are designed to specifically disrupt BIG3 binding, may demonstrate excellent therapeutic indices with minimal off-target effects. Intracellular protein-protein interactions have been difficult to target with small molecules or synthetic peptide inhibitors, regardless of their ability to regulate many signalling networks. However, it has been reported that the Nutlins, selective small molecule antagonists of the MDM2-p53 interaction, possess in vitro and in vivo anti-tumour activity via the reactivation of $\mathrm{p} 53$ tumour suppressive activity ${ }^{48}$. In fact, as the inhibitory effect of ERAP was maintained for only $24 \mathrm{~h}$ (Supplementary Fig. S5b), it will be necessary to improve upon its pharmacologic properties using chemical synthetic approaches, such as hydrocarbon stapling methods ${ }^{49,50}$, or to screen selective small molecule antagonists targeting the BIG3PHB2 interaction.

In conclusion, targeting the BIG3-PHB2 interaction represents a potential new treatment avenue for ER $\alpha$-positive breast cancer patients. This new approach could be an important supplement therapy and may provide mechanistic insight into the molecular basis of $E R \alpha$-signalling networks in breast carcinogenesis. Thus, combining endocrine treatment with these new targeted therapies is a promising approach for improving the current treatment strategies and overcoming endocrine resistance, and should be investigated in future preclinical and clinical studies.

\section{Methods}

ER $\boldsymbol{\alpha}$ antibody. The anti-ER $\alpha$ (clone AER314) antibody was purchased from Thermo Fisher Scientific (Fremont, CA). This antibody specifically recognizes undigested $\mathrm{ER} \alpha$ and is equivalent to an anti-ER $\alpha$ antibody (SP-1) ${ }^{34}$, which is widely used for immunohistochemical analysis (Supplementary Fig. S8).

Immunoblot analyses. Cells were lysed with lysis buffer (50 mM Tris- $\mathrm{HCl}, \mathrm{pH} 8.0$; $150 \mathrm{mM} \mathrm{NaCl}, 0.1 \% \mathrm{NP}-40,0.5 \%$ CHAPS) containing $0.1 \%$ protease inhibitor cocktail III (Calbiochem, San Diego, CA). The lysates were electrophoretically separated, blotted onto a nitrocellulose membrane and blocked with $4 \%$ BlockAce solution (Dainippon Pharmaceutical, Osaka, Japan) for $1 \mathrm{~h}$. The blots were then incubated with antibodies against the following proteins: BIG3 (ref. 21) (1:200); PHB2 (1:500), NcoR (1:500) and ER $\alpha$ (phospho Y537; 1:500; Abcam, Cambridge, UK); SRC-1 (128E7; 1:500), Shc (1:500), $\alpha / \beta$-tubulin $(1: 1,000)$, Akt $(1: 1,000)$, phospho-Akt (S473; 587F11; 1:1,000), p44/42 MAPK (1:500), phospho-p44/42 MAPK (T202/Y204; 1:500) and phospho-ER $\alpha$ (S104/S106; 1:500; Cell Signaling Technology, Danvers, MA); HDAC1 (H-11; 1:500), IGF-1R $\beta$ (1:500), PI3-kinase $\mathrm{p} 85 \alpha$ (U13; 1:500), phospho-ER $\alpha(\mathrm{S} 118 ; 1: 500)$, phospho-ER $\alpha(\mathrm{S} 167 ; 1: 500)$ and laminin B1 (1:100; Santa Cruz Biotechnology, Santa Cruz, CA); phospho-ER $\alpha$ (S305; 1:500; Millipore, Billerica, MA); phosphotyrosine (1:500; Zymed, San Francisco, CA); $\beta$-actin (AC-15; 1:5,000) and FLAG-tag M2 (1:5,000; Sigma, St Louis, MO); and HA-tag (1:3,000; Roche, Mannheim, Germany). After incubation with an horseradish peroxidase-conjugated secondary antibody (Santa Cruz Biotechnology, dilution 1:5,000) or monoclonal anti-rabbit immunoglobulins-peroxidase antibody (RG-16, Sigma, dilition 1:5,000) for $1 \mathrm{~h}$, the blots were developed with an enhanced chemiluminescence system (GE Healthcare, Buckinghamshire, UK) and were scanned using an Image Reader LAS-3000 mini (Fujifilm, Tokyo, Japan). All experiments were performed more than three times in triplicate. Finally, the phosphorylation levels of IGF-1R $\beta$, Shc, PI3K, Akt, p42/44 MAPK and ER $\alpha$ were assessed through densitometric analysis of immunoblot results using an Image Reader LAS-3000 mini ${ }^{51}$. Full-length images of immunoblots are shown in Supplementary Fig. S9.

Immunoprecipitation. The cells were lysed with $0.1 \% \mathrm{NP}-40$ lysis buffer as described above. The cell lysates were precleared with normal IgG and rec-Protein $\mathrm{G}$ Sepharose $4 \mathrm{~B}$ (Zymed) at $4{ }^{\circ} \mathrm{C}$ for $3 \mathrm{~h}$. Then, the supernatants were incubated with antibodies against BIG3 $(5 \mu \mathrm{g})$, PHB2 $(5 \mu \mathrm{g})$ and $\mathrm{ER} \alpha(5 \mu \mathrm{g})$ at $4{ }^{\circ} \mathrm{C}$ for $12 \mathrm{~h}$. Next, the antigen-antibody complexes were precipitated with rec-Protein $G$ Sepharose $4 \mathrm{~B}$ at $4{ }^{\circ} \mathrm{C}$ for $1 \mathrm{~h}$. Immunoprecipitated protein complexes were washed three times with lysis buffer and separated using SDS-PAGE. Immunoblot analyses were performed as described above.

Identification of the PHB2-binding regions in BIG3 protein. To determine the PHB2-binding region in BIG3, we cloned five different constructs corresponding to partial BIG3 sequences $\left(\mathrm{BIG}_{1-434}, \mathrm{BIG}_{435-2,177}, \mathrm{BIG}_{1,468-2,177}, \mathrm{BIG}_{1-100}\right.$ and BIG3 $_{1-250}$ ) into an amino-terminal FLAG-tagged pCAGGS vector. COS-7 cells were individually cotransfected with a BIG3 vector and HA-PHB2 using the FuGENE6 transfection reagent (Roche). At $48 \mathrm{~h}$ after transfection, the cells were lysed with $0.1 \%$ NP-40 lysis buffer. The lysates were precleared for $3 \mathrm{~h}$ at $4{ }^{\circ} \mathrm{C}$ and then incubated with anti-FLAG M2 agarose (Sigma) for $12 \mathrm{~h}$ at $4{ }^{\circ} \mathrm{C}$. Next, the immunoprecipitated proteins or intact cell lysates were electrophoresed and blotted 
onto nitrocellulose. Finally, the blots were incubated with antibodies against FLAG M2 or HA-tag.

Interaction site and structure prediction. BIG3 and PHB2 interaction sites were predicted using PSIVER. PSIVER is a computational method to predict residues that bind to other proteins using only sequence features (position-specific scoring matrix and predicted accessibility). The method uses the Naive Bayes classifier with kernel density estimation and was shown to outperform existing servers available on the Internet. The default threshold of 0.390 was used in this study. Structure prediction was performed using FUGUE ${ }^{52}$ and PSIPRED ${ }^{53}$. A model of the putative PHB2-binding helix of BIG3 (residues 157-174) was built using MODELLER $^{54}$ based on the TIP120 protein $^{55}$ as a template.

BIG3-PHB2 interaction inhibition by ERAP. The 13 amino acid peptides derived from the PHB2-binding domain of BIG3 (codons 165-177) were covalently linked at the $\mathrm{NH}_{2}$ terminus to a membrane transducing 11 polyarginine sequence $(11 \mathrm{R})$ to construct the ERAP peptide. Negative control peptides, scrERAP and mtERAP, were also synthesized. To examine the effects of ERAP on inhibition of BIG3PHB2 complex formation, MCF-7 cells were treated with $10 \mathrm{nM}$ E2 $\pm 10 \mu \mathrm{M}$ ERAP. BIG3-PHB2 interactions were assessed using co-immunoprecipitation followed by immunoblotting, as described above.

Nuclear/cytoplasmic fractionation. MCF-7 cells were treated as described above, and nuclear and cytoplasmic/plasma membrane fractions were prepared using the NE-PER nuclear and cytoplasmic extraction reagent (Thermo Fisher Scientific) according to the manufacturer's instructions. $\alpha / \beta$-Tubulin and laminin B were used as loading controls for the cytoplasmic and nuclear fractions, respectively.

Cell proliferation assay. Cell proliferation assays were performed using the Cell Counting Kit-8 (Dojindo, Kumamoto, Japan). The cells were plated in 48-well plates at $2 \times 10^{4}$ cells per well and maintained at $37^{\circ} \mathrm{C}$. At the indicated time points, a 1:10 dilution of the Cell Counting Kit- 8 solution was added (into three replicate wells) and incubated for $1 \mathrm{~h}$. Then, the absorbance at $450 \mathrm{~nm}$ was measured to calculate the number of vital cells per well. The data represent the mean \pm s.e.m. of three independent experiments.

In vivo tumour growth inhibition. Each suspension $\left(1 \times 10^{7}\right.$ cells per mouse $)$ of KPL-3C cells, MCF-7 cells, T47D cells, TAM-R MCF-7 cells or TAM-R T47D cells was mixed with an equal volume of Matrigel (BD, Franklin Lakes, NJ) and injected $(200 \mu \mathrm{l}$ total) into the mammary fat pads of 6-week-old female BALB/c nude mice (Charles River Laboratories, Tokyo, Japan). The mice were housed in a pathogenfree isolation facility with a 12-h light/dark cycle, and were fed rodent chow and water ad libitum. The tumours developed over a period of 1 week, reaching sizes of $\sim 100 \mathrm{~mm}^{3}$ (calculated as $1 / 2 \times\left(\right.$ width $\times$ length $\left.\left.^{2}\right)\right)$. For KPL-3C orthotropic xenograft experiments, the mice were randomized into eight treatment groups (five animals per group): (1) no treatment; (2) $6 \mu \mathrm{g}$ per day E2; (3-5) E2 +3.5, 7 or $14 \mathrm{mg} \mathrm{kg}^{-1}$ per day ERAP; (6) E2 $+14 \mathrm{mg} \mathrm{kg}^{-1}$ per day scrERAP; (7) E2 +4 $\mathrm{mg} \mathrm{kg}-1$ per day tamoxifen; and (8) $\mathrm{E} 2+4 \mathrm{mg} \mathrm{kg}^{-1}$ per day tamoxifen +14 $\mathrm{mg} \mathrm{kg}{ }^{-1}$ per day ERAP. For the MCF-7 orthotopic xenograft, the mice were randomized into three treatment groups: (1) $6 \mu \mathrm{g}$ per day E2; (2) E2 $+14 \mathrm{mg} \mathrm{kg}^{-1}$ per day ERAP; and (3) E2 $+14 \mathrm{mg} \mathrm{kg}^{-1}$ per day scrERAP. For the T47D orthotopic xenograft, the mice were randomized into five treatment groups: (1) no treatment; (2) $6 \mu \mathrm{g}$ per day E2; (3) E2 $+14 \mathrm{mg} \mathrm{kg}^{-1}$ per day ERAP; (4) E2 +3.7 $\mu \mathrm{gg}^{-1}$ per day tamoxifen; and (5) E2 + ERAP + tamoxifen. For TAM-R MCF-7 orthotropic xenograft, the mice were randomized into four treatment groups: (1) no treatment; (2) $37 \mu \mathrm{g} \mathrm{kg}^{-1}$ per day tamoxifen; (2) $6 \mu \mathrm{g}$ per day E2 + tamoxifen; and $(3,4) \mathrm{E} 2+$ tamoxifen +7 or $14 \mathrm{mg} \mathrm{kg}^{-1}$ per day ERAP. For TAM-R T47D orthotopic xenografts, the mice were randomized into four treatment groups: (1) no treatment; (2) $3.7 \mu \mathrm{g} \mathrm{kg}^{-1}$ per day tamoxifen; (3) $6 \mu \mathrm{g}$ per day E2 + tamoxifen; and (4) E2 + tamoxifen $+14 \mathrm{mg} \mathrm{kg}^{-1}$ per day ERAP. E2 was delivered via the application of a solution to the skin at the neck; the other treatments were delivered via intraperitoneal injection. Tumour volume was measured with calipers for 2 weeks, after which time the animals were killed, and the tumours were excised and frozen in liquid nitrogen. All experiments were performed in accordance with the guidelines of the animal facility at the University of Tokushima. For evaluations of the inhibitory effects of ERAP on tumour expression of ER $\alpha$ target genes using real-time PCR, the data are expressed as the fold increase in gene expression over the no treatment group (set at 1.0) and represent the mean \pm s.e.m. of each group (five mice). In addition, for evaluations of the effects of ERAP on the phosphorylation of Akt, p42/44 MAPK and ER $\alpha$ proteins in tumours, each tumour lysates (three to four mice per group) was immunoblotted.

Statistical analyses. A Student's $t$-test was used to determine the significance of differences among the experimental groups. Values of $P<0.05$ were considered significant.

The other methods are described in Supplementary Information.

\section{References}

1. McCracken, M. et al. Cancer incidence, mortality, and associated risk factors among Asian Americans of Chinese, Filipino, Vietnamese, Korean, and Japanese Ethnicities. CA Cancer J. Clin. 57, 190-205 (2007).

2. Jemal, A. et al. Cancer statistics, 2006. CA Cancer J. Clin. 56, 106-130 (2006)

3. Yager, J. D. \& Davidson, N. E. Estrogen carcinogenesis in breast cancer. N. Engl. J. Med. 354, 270-282 (2006).

4. Berry, D. A. et al. Effect of screening and adjuvant therapy on mortality from breast cancer. N. Engl. J. Med. 353, 1784-1792 (2005).

5. Green, S. \& Chambon, P. Nuclear receptors enhance our understanding of transcription regulation. Trends Genet. 4, 309-314 (2000).

6. Kahlert, S. et al. Estrogen receptor rapidly activates the IGF-1 receptor pathway. J. Biol. Chem. 275, 18447-18453 (2000).

7. Simoncini, T. et al. Interaction of oestrogen receptor with the regulatory subunit of phosphatidylinositol-3-OH kinase. Nature 407, 538-541 (2000).

8. Castoria, G. et al. PI3-kinase in concert with Src promotes the S-phase entry of oestradiol-stimulated MCF-7 cells. EMBO J. 20, 6050-6059 (2001).

9. Song, R. X. et al. The role of Shc and insulin-like growth factor 1 receptor in mediating the translocation of estrogen receptor $\alpha$ to the plasma membrane. Proc. Natl Acad. Sci. USA 101, 2076-2081 (2004).

10. Osborne, C. K. \& Schiff, R. Estrogen-receptor biology: continuing progress and therapeutic implications. J. Clin. Oncol. 23, 1616-1622 (2005).

11. Johnston, S. R. New strategies in estrogen receptor-positive breast cancer. Clin. Cancer Res. 16, 1979-1987 (2010).

12. Fisher, B. et al. Tamoxifen for prevention of breast cancer: report of the National Surgical Adjuvant Breast and Bowel Project P-1 Study. J. Natl Cancer Inst. 97, 1652-1662 (2005).

13. Jordan, V. C. Tamoxifen: a most unlikely pioneering medicine. Nat. Rev. Drug Discov. 2, 205-213 (2003).

14. Clarke, R., Leonessa, F., Welch, J. N. \& Skaar, T. C. Cellular and molecular pharmacology of antiestrogen action and resistance. Pharmacol. Rev. 53, 25-71 (2001).

15. Fisher, B., Dignam, J., Bryant, J. \& Wolmark, N. Five versus more than five years of tamoxifen for lymph node-negative breast cancer: updated findings from the National Surgical Adjuvant Breast and Bowel Project B-14 randomized trial. J. Natl Cancer Inst. 93, 684-690 (2001).

16. Ring, A. \& Dowsett, M. Mechanisms of tamoxifen resistance. Endocr. Relat. Cancer 11, 643-658 (2004).

17. Leary, A. F., Sirohi, B. \& Johnston, S. R. Clinical trials update: endocrine and biological therapy combinations in the treatment of breast cancer. Breast Cancer Res. 9, 112 (2007).

18. Leary, A. F. et al. Lapatinib restores hormone sensitivity with differential effects on estrogen receptor signaling in cell models of human epidermal growth factor receptor 2-negative breast cancer with acquired endocrine resistance. Clin. Cancer Res. 16, 1486-1497 (2010).

19. Osborne, C. K. et al. Gefitinib or placebo in combination with tamoxifen in patients with hormone receptor-positive metastatic breast cancer: a randomized phase II study. Clin. Cancer Res. 17, 1147-1159 (2011).

20. Nishidate, T. et al. Genome-wide gene-expression profiles of breast-cancer cells purified with laser microbeam microdissection: identification of genes associated with progression and metastasis. Int. J. Oncol. 25, 797-819 (2004).

21. Kim, J. W. et al. Activation of an estrogen/estrogen receptor signaling by BIG3 through its inhibitory effect on nuclear transport of PHB2/REA in breast cancer. Cancer Sci. 100, 1468-1478 (2009).

22. Montano, M. M. et al. An estrogen receptor-selective coregulator that potentiates the effectiveness of antiestrogens and represses the activity of estrogens. Proc. Natl Acad. Sci. USA 96, 6947-6952 (1999).

23. Delage-Mourroux, R. et al. Analysis of estrogen receptor interaction with a repressor of estrogen receptor activity (REA) and the regulation of estrogen receptor transcriptional activity by REA. J. Biol. Chem. 275, 35848-35856 (2000).

24. Murakami, Y. \& Mizuguchi, K. Applying the naive bayes classifier with kernel density estimation to the prediction of protein-protein interaction sites. Bioinformatics 26, 1841-1848 (2010).

25. Klinge, C. M. Estrogen receptor interaction with estrogen response elements. Nucleic Acids Res. 29, 2905-2919 (2001).

26. Kushner, P. L. et al. Estrogen receptor pathways to AP-1. J. Steroid Biochem. Mol. Biol. 74, 311-317 (2000).

27. Kurtev, V. et al. Transcriptional regulation by the repressor of estrogen receptor activity via recruitment of histone deacetylases. J. Biol. Chem. 279, 24834-24843 (2004).

28. Varlakhabova, N., Snyder, C., Jose, S., Hahm, J. B. \& Privalsky, M. L. Estrogen receptors recruit SMRT and $\mathrm{N}-\mathrm{CoR}$ corepressors through newly recognized contacts between the corepressor $\mathrm{N}$ terminus and the receptor DNA binding domain. Mol. Cell Biol. 30, 1434-1445 (2010).

29. Brown, A. M., Jeltsch, J. M., Roberts, M. \& Chambon, P. Activation of pS2 gene transcription is a primary response to estrogen in the human breast cancer cell line MCF-7. Proc. Natl Acad. Sci. USA 81, 6344-6348 (1984) 
30. Altucci, L. et al. 17beta-Estradiol induces cyclin D1 gene transcription, p36D1p34cdk4 complex activation and p105Rb phosphorylation during mitogenic stimulation of G (1)-arrested human breast cancer cells. Oncogene 12, 2315-2324 (1996)

31. Dubik, D., Dembinski, T. C. \& Shiu, R. P. Stimulation of c-myc oncogene expression associated with estrogen-induced proliferation of human breast cancer cells. Cancer Res. 47, 6517-6521 (1987).

32. Jin, V. X., Rabinovich, A., Squazzo, S. L., Green, R. \& Farnham, P. J. A computational genomics approach to identify cisregulatory modules from chromatin immunoprecipitation microarray data - a case study using E2F1. Genome Res. 16, 1585-1595 (2006).

33. Yu, W. C., Leung, B. S. \& Gao, Y. L. Effects of 17 beta-estradiol on progesterone receptors and the uptake of thymidine in human breast cancer cell line CAMA-1. Cancer Res. 41, 5004-5009 (1981).

34. Welsh, A. W. et al. Cytoplasmic estrogen receptor in breast cancer. Clin. Cancer Res. 18, 118-126 (2012).

35. Mintz, P. J. et al. The phosphorylated membrane estrogen receptor and cytoplasmic signaling and apoptosis proteins in human breast cancer. Cancer 113, 1489-1495 (2008).

36. Murphy, L. C., Seekallu, S. V. \& Watson, P. H. Clinical significance of estrogen receptor phosphorylation. Endocr. Relat. Cancer 18, R1-R14 (2011).

37. Lannigan, D. A. Estrogen receptor phosphorylation. Steroids 68, 1-9 (2008).

38. Chen, D. et al. Activation of estrogen receptor alpha by S118 phosphorylation involves a ligand-dependent interaction with TFIIH and participation of CDK7. Mol. Cell 6, 127-137 (2000).

39. Chen, D., Pace, P. E., Coombes, R. C. \& Ali, S. Phosphorylation of human estrogen receptor alpha by protein kinase A regulates dimerization. Mol. Cell Biol. 19, 1002-1015 (1999).

40. Joel, P. B. et al. pp90rsk1 regulates estrogen receptor-mediated transcription through phosphorylation of Ser-167. Mol. Cell Biol. 18, 1978-1984 (1998).

41. Rogatsky, I., Trowbridge, J. M. \& Garabedian, M. J. Potentiation of human estrogen receptor alpha transcriptional activation through phosphorylation of serines 104 and 106 by the cyclin A-CDK2 complex. J. Biol. Chem. 274, 22296-22302 (1999).

42. Arnold, S. F., Melamed, M., Vorojeikina, D. P., Notides, A. C. \& Sasson, S Estradiol-binding mechanism and binding capacity of the human estrogen receptor is regulated by tyrosine phosphorylation. Mol. Endocrinol. 11, 48-53 (1997).

43. Wang, R. A., Mazumdar, A., Vadlamudi, R. K. \& Kumar, R. P21-activated kinase-1 phosphorylates and transactivates estrogen receptor-alpha and promotes hyperplasia in mammary epithelium. EMBO J. 21, 5437-5447 (2001).

44. Taylor, I. W., Hodson, P., Green, M. D. \& Sutherland, R. L. Effects of tamoxifen on cell cycle progression of synchronous MCF-7 human mammary carcinoma cells. Cancer Res. 43, 4007-4010 (1983).

45. Oyama, M. et al. Integrated quantitative analysis of the phosphoproteome and transcriptome in tamoxifen-resistant breast cancer. J. Biol. Chem. 286, 818-829 (2011).

46. Fagan, D. H., Uselman, R. R., Sachdev, D. \& Yee, D. Acquired resistance to tamoxifen is associated with loss of the type I insulin-like growth factor receptor: implications for breast cancer treatment. Cancer Res. 72, 3372-3380 (2012).

47. Sato, T. et al. The human prohibitin (PHB) gene family and its somatic mutations in human tumours. Genomics 17, 762-764 (1993).

48. Vassilev, L. T. et al. In vivo activation of the p53 pathway by small-molecule antagonists of MDM2. Science 303, 844-848 (2004).

49. Walensky, L. D. et al. Activation of apoptosis in vivo by a hydrocarbon-stapled BH3 helix. Science 305, 1466-1470 (2004).

50. Moellering, R. E. et al. Direct inhibition of the NOTCH transcription factor complex. Nature 462, 182-188 (2009).
51. Abramoff, M. D., Magelhaes, P. J. \& Ram, S. J. Image processing with Image J. Biophotonics Int. 11, 36-42 (2004).

52. Shi, J., Blundell, T. L. \& Mizuguchi, K. FUGUE: sequence-structure homology recognition using environment-specific substitution tables and structuredependent gap penalties. J. Mol. Biol. 310, 243-257 (2001).

53. McGuffin, L. J., Bryson, K. \& Jones, D. T. The PSIPRED protein structure prediction server. Bioinformatics 16, 404-405 (2000).

54. Eswar, N., Eramian, D., Webb, B., Shen, M. Y. \& Sali, A. Protein structure modeling with MODELLER. Methods Mol. Biol. 426, 145-159 (2008).

55. Goldenberg, S. J. et al. Structure of the Cand1-Cul1-Roc1 complex reveals regulatory mechanisms for the assembly of the multisubunit cullin-dependent ubiquitin ligases. Cell 119, 517-528 (2004).

\section{Acknowledgements}

We thank Drs Douglas Yee and Dedra Fagan (University of Minnesota) for the gift of the TAM-R T47D cell line; Dr Takao Yamori (Cancer Chemotherapy Centre, Japanese Foundation for Cancer Research) for the gift of the HBC4 breast cancer cell line; Dr Junichi Kurebayashi (Kawasaki Medical School) for the gifts of the KPL-3C and KPL-1 breast cancer cell lines; Drs Kazuhiro Ikeda and Satoshi Inoue (Saitama Medical University) for the gifts of the TAM-R-MCF-7 cells; and Drs Tomoya Fukawa and Kazuma Kiyotani (The University of Tokushima) for helpful discussions. We acknowledge support from the Takeda Science Foundation, Naito Foundation, the Mochida Memorial Foundation for Medical and Pharmaceutical Research, the Takamatsunomiya Princess Memorial Foundation, Project Future of Relay For Life Japan and a Grant-in-Aid for Scientific Research on Innovative Areas from MEXT (T.K., 2011-2012) (23134504), (T.K., 2013-2014)(25134712).

\section{Author contributions}

T.Y. performed all experiments. M.K. performed in vivo experiments. T.M. performed the affinity purification of BIG3 antibody and was involved in functional analysis. Y.-A.C., Y.M. and K.M. performed in silico analysis for prediction of BIG3-PHB2 interaction. E.M. and T.I. performed purification of recombinant PHB2 protein. M.A. identified BIG3 through the expression profiling analysis. R.Y., S.I., S.M. and Y.M. discussed the interpretation of ER-signalling pathway data. M.S. collected breast cancer specimens. Y.N. supported the preparation of the final version of the manuscript. T.K. was involved in the conception and design of all studies, interpretation of data and preparing the draft and final version of the manuscript. All authors read and approved the final manuscript. M.K. and T.M. contributed equally to this work.

\section{Additional information}

Supplementary Information accompanies this paper at http://www.nature.com/ naturecommunications

Competing financial interests: Toyomasa Katagiri is an external board member of OncoTherapy Science, Inc. The remaining authors declare no competing financial interest.

Reprints and permission information is available online at http://npg.nature.com/ reprintsandpermissions/

How to cite this article: Yoshimaru, T. et al. Targeting BIG3-PHB2 interaction to overcome tamoxifen resistance in breast cancer cells. Nat. Commun. 4:2443 doi: $10.1038 /$ ncomms3443 (2013).

(c) (1) $\odot$ This work is licensed under a Creative Commons Attributioncc) NonCommercial-NoDerivs 3.0 Unported License. To view a copy of this license, visit http://creativecommons.org/licenses/by-nc-nd/3.0/ 